[The following Paper was read at Third and Fourth Meetings, 8th January and 12th February 1897.]

\title{
On the Geometrical Representation of Elliptic Integrals of the First Kind.
}

By Alex. Morgan, M.A., B.Sc.

I.

When an expression has to be integrated which contains the square root of a rational function of the first or second degree, the integral can be expressed in terms of the ordinary algebraic functions or the elementary transcendental functions, viz., exponential and circular. But when the polynomial under the radical is higher than the second degree its integral in general can only be expressed by means of transcendentals of a higher kind. The particular case in which the expression under the square root is a cubic or quartic gives rise to a class of definite integrals called Elliptic Integrals, because by means of them, as we shall see, we can express the length of the arc of an ellipse or other central conic.

Legendre * considered the general elliptic integral $\int \frac{\mathrm{P} d x}{\sqrt{\mathrm{X}}}$,

where $\mathrm{P}$ is any rational function whatever of $x$, and $\mathrm{X}$ is a positive rational integral quartic function of $x$ with real coefficients, and he showed that by partial integrations and by transformations this integral could be resolved into an algebraic part together with transcendentals always of three types $\dagger$, viz.,

$\int \frac{d x}{\sqrt{1-x^{2} \cdot 1-k^{2} x^{2}}} \int \frac{x^{2} d x}{\sqrt{1-x^{2} \cdot 1-k^{2} x^{2}}} \int \frac{d x}{\left(1+n x^{2}\right) \sqrt{1-x^{2} \cdot 1-k^{2} x^{2}}}$,

where $k$ is $+{ }^{* e}$ and less than 1 , and $n$ is real or imaginary.

Without altering the type of the second integral we may write it

$$
\int \frac{\left(1-k^{2} x^{2}\right) d x}{\sqrt{1-x^{2} \cdot 1-k^{2} x^{2}}} \text { or } \int \sqrt{\frac{1-k^{2} x^{2}}{1-x^{2}}} d x
$$

* Traité de Fonctions Elliptiques, t. I., Chap. iii., iv. and v. $\dagger c f$. CaYley's Elliptic Functions, $\$ 1$. 
Putting $x=\sin \phi$, we thus have three kinds of elliptic integrals :

First kind, $\quad \mathbf{F}(k, \phi)=\int_{0}^{\phi} \frac{d \phi}{\sqrt{1-k^{2} \sin ^{2} \phi}}$

Second kind, $\quad \mathbf{E}(k, \phi) \quad=\int_{0}^{\phi} \sqrt{1-k^{2} \sin ^{2} \phi} d \phi$

Third kind, $\quad \Pi(n, k, \phi)=\int_{0}^{\phi} \frac{d \phi}{\left(1+n \sin ^{2} \phi\right) \sqrt{1-k^{2} \sin ^{2} \phi}}$

$\phi$ is called the amplitude, and is a real angle, $k$ the modulus, and $n$ the parameter.

The arc of an ellipse can be represented by an elliptic integral of the second kind. Thus in the ellipse

$$
\frac{x^{2}}{a^{2}}+\frac{y^{2}}{b^{2}}=1
$$

if we put

$$
\begin{aligned}
x & =a \sin \phi, y=b \cos \phi \\
d s^{2} & =d x^{2}+d y^{2} \\
& =\left(a^{2} \cos ^{2} \phi+b^{2} \sin ^{2} \phi\right) d \phi^{2} \\
& =\left[a^{2}-\left(a^{2}-b^{2}\right) \sin ^{2} \phi\right] d \phi^{2} \\
& =a^{2}\left(1-e^{2} \sin ^{2} \phi\right) d \phi^{2} \\
\therefore s & =a \int \sqrt{1-e^{2} \sin ^{2} \phi} d \phi \\
& =a \mathrm{E}(e, \phi),
\end{aligned}
$$

the arc being measured from the extremity of the minor axis.

It is thus very easy to find a curve whose arc will represent an elliptic integral of the second kind, but it has always been a difficult problem to give a complete geometrical representation of integrals of the first kind.*

\section{Historical Note.}

The name elliptic functions is somewhat of a misnomer, as the whole theory of these functions is based on the first elliptic integral $\mathrm{F}(k, \phi)$ which can not be represented by an arc of an ellipse.

* For references to the numerous attempts to solve the problem, see Note V. in the Appendix of Müller's edition of Enneper's Elliptische Functionen (Halle, 1890). 
It was LwarNdRE (MÉmoire sur les transcendantes elliptiques, 1793 : Exxercices du Calcul Intégral sur divers ordres de Transcendantes et sur les Quadratures, 3 vols. 1811-1819 ; and Traite des Fonctions Elliptiques, 3 vols. 1825-32) who discovered most of the important properties of the new functions, and invented a notation for them ; but the founder of the modern theory of elliptic functions may fairly enough be said to be ABEL (his earlier Memoirs on the subjeet appeared in Crelle's Journal, 1826-9. They are collected in CEuvres Completes de N.H. Abel par B. Holmboe, 1839. His great Memoir on Transcendental Functions is published by the French Academy in Mémoires des Savants Etrangers, t. vii., 1841. The most recent edition of his works is $N . H$. Abel. Tableau de sa vie et de son action scientifique. Par C. A. Bjerknes, 1885).

About 1823 ABEL pointed out that in

$$
F(k, \phi)=\int_{0}^{\phi}\left(1-k^{2} \sin ^{2} \phi\right)-\frac{1}{2} d \phi
$$

$F(k, \phi)$ is of the nature of an inverse function, and that if we put $u=F(k, \phi)$ then we should study the properties of the amplitude $\phi$ as a function of $u$, and not $u$ as a function of $\phi$. Legendre laid great stress on the elliptic integrals, and tried to deduce the properties of elliptic functions from them, but Abel pointed out that by following Legendre's method mathematicians were making the same mistake as if they had tried to deduce the theorems of trigonometry by studying the properties of the inverse circular functions as deduced from the circular integrals. It is Abel's idea of the inversion of the first elliptic integral, and his discovery of the double periodicity of elliptic functions that have led to the wonderful recent advances in the theory of elliptic and higher transcendentals in Germany and France.

It is interesting to trace the genesis of elliptic functions out of the early attempts of geometers to rectify the ellipse.

Maclaurin ( $A$ Treatise of Fluxions, Edinburgh 1742) and D'AlkmBkRT (Des Difféventielles qui se rapportent à la rectification de l'ellipse ou de l'hyperbole in the Histoire de l'Acad. de Berlin, 1746) seem to have been the first to study integrals which could be expressed by the arcs of an ellipse or hyperbola. They found a great many such integrals, but their results were disjointed.

Next, Fagnano (Produzioni Matenatiche Del Mrarchese Giulio Carlo De' Toschi Di Fagnano. 2 vols. Pesaro 1750) proved that in any given ellipse or hyperbola we can in an infinite number of ways find two arcs of which the difference is expressible by an algebraio quantity.

It was EvLER, however, who first tried to develop a general theory out of those scattered theorems. He clearly foresaw that with a suitable notation a new kind of functions, founded on the properties of the arcs of ellipses, would arise which would become as general and as important in the higher analysis as were the circular and logarithmic functions. At vol. $X$. p. 4 of Novi Commentarii Acad. Sc. Petropoli (1761), Euler says "Imprimis autem hic idoneus signandi modus desiderari videtur, cujus ope arcus elliptici aeque commode in calculo exprimi queant, ac jam logarithmi et arcus circulares ad insigne Analyseos per idones signa in calculum sint introducti. Talia signa novam quandam calculi speciem suppeditabunt. . . . . ." Wo shall see 
that this passage had afterwards a most important effect on the work of Legendre.

Also Euler (Novi Com., Vols. vi. and vii., 1761) discovered the method of integrating by algebraical funetions the differential equation now known as Euler's Equation, viz.,

$$
\mathrm{X}^{-\frac{1}{d}} d x+\mathrm{Y}^{-\frac{1}{2}} d y=0
$$

where $\mathrm{X}$ is a quartic function of $x$ and $\mathrm{Y}$ is the same function of $y$, say

$$
\begin{aligned}
& \mathrm{X}=a x^{4}+b x^{3}+c x^{2}+e x+f \\
& \mathrm{Y}=a y^{4}+b y^{3}+c y^{2}+e y+f .
\end{aligned}
$$

The integral is $\quad\left(\frac{\mathrm{X}^{\frac{1}{2}}-\mathrm{Y}^{\frac{1}{2}}}{x-y}\right)^{2}=\mathrm{C}+a(x+y)^{2}+b(x+y)$.

He thus found the complete algebraical integral of a differential equation composed of two similar terms, whose integrals taken separately are not algebraic but only expressible by the arcs of an ellipse or other conic section.

JoHN LANDEN (Philosophical I'ransactions, 1775 ; Mathematical Memoirs, 1780) proved that every arc of a hyperbola can be expressed in terms of the arcs of two ellipses. This theorem was an important step in the simplification of the theory of such arcs.

LFGENDRE in 1786 published the first of his investigations in connection with the subject. In that year there appeared his Mémoire sur les intégrations par d'arcs d'ellipse in Mém. de l'Acad. des Sciences de Paris. Among other things he proved that in an infinite series of ellipses formed according to the same law the rectification of one of the ellipses can be reduced to that of two others chosen at will from the series.

Not, however, until his attention was arrested by Euler's discovery of 1761 and prediction of 1764 did Legendre perceive the way in which the new functions were to be attacked with success. He was led by Euler's equation to examine all the transcendentals contained in $\int \frac{P d x}{\sqrt{X}}$ where $P$ is any rational function of $x$, and $\mathrm{X}$ a rational function of $x$ of the fourth degree. He classified the integrals included under this general form into three kinds, and developed a notation and theory for the reduced integrals as had been desiderated by Euler in 1764. These results are contained in his Mémoire sur les Transcendantes elliptiques, 1793. All the investigations of Legendre were afterwards collected and published in his Exercices du Calcul Intégral etc., and his Traité des Fonctions Elliptiques already mentioned.

JACOBI (Fundamenta Nova Theorio Functionum Ellipticartm, 1829, and Memoirs in Crelle's Journal, 1828-1858) carried out Abel's idea of the inversion of the first elliptic integral and introduced a notation to take the place of Legendre's. He took $F(k, \phi)$ as the independent variable and put $u$ for it, calling $\phi$ the amplitude of $u$ or shortly $\phi=\operatorname{am} u$. Then $\sin \phi, \cos \phi$ and $\Delta \phi\left(=\sqrt{1-k^{2} \sin ^{2} \phi}\right)$ were the sine, cosine, and $\Delta$ of the amplitude of $u$, or as he wrote them $\sin \operatorname{am} u, \cos \mathrm{am} u, \Delta \operatorname{am} u$. In the Fundamenta Nova he developed with great elegance the properties of these three elliptic functions 
of $u$. He also changed the meanings of Legendre's symbols $E$ and II for the elliptic integrals of the second and third kinds into those denoted by the equations

$$
\mathbf{E} u=\int_{0}^{u} \operatorname{dn} u d u, \quad \Pi(u, a)=\int_{0}^{u} \frac{k^{2} \operatorname{sm} a \operatorname{cn} a \operatorname{dn} a \operatorname{sn}^{2} u}{1-k^{2} \operatorname{sn}^{2} u \operatorname{sn}^{2} a} d u
$$

He introduced new functions zeta, theta, and eta defined as follows :-

$$
\begin{aligned}
& \mathbf{Z} u=\mathbf{E} u-\frac{u \mathrm{E}}{\mathbf{K}}, \quad \log \frac{\Theta u}{\Theta o}=\int_{0}^{u} \mathrm{Z} u d u, \text { or } \Theta u=\theta 0 \exp \cdot \int_{0}^{u} \mathrm{Z} u d u \\
& \mathbf{H} u=\sqrt{ } k \cdot \Theta u . \operatorname{sn} u .
\end{aligned}
$$

It was Gudkrmann (Theorie der Modular Functionen in Crelle's Journal, vol. xviii. p. 12) who proposed the abbreviations sn, cn, dn for sin am, cos am, $\Delta \mathrm{am}$, and Dr Glatsher (Messenger of Math., vol. xi. p. 86) who introduced the notation

$\begin{array}{llllll} & \operatorname{ns} u & \operatorname{nc} u & \operatorname{nd} u & \operatorname{sc} u & \operatorname{cd} u \text { etc. } \\ \text { for } & \frac{1}{\operatorname{sn} u} & \frac{1}{\operatorname{cn} u} & \frac{1}{\operatorname{dn} u} & \frac{\operatorname{sn} u}{\operatorname{cn} u} & \frac{\operatorname{cn} u}{\operatorname{dn} u} \text { etc. }\end{array}$

In recent times no worker has done so much to develop the theory as Werkestrass. While Jacobi's functions sn $u$, cn $u, \mathrm{dn} u$ are recognised as valuable for numerical work, it is granted on all hands that Weierstrass's functions

$$
\sigma u=u \Pi_{*}\left(1-\frac{u}{w}\right) e^{\frac{u}{v v}+\frac{1}{2} \frac{u^{2}}{w^{2}}}
$$

$\left(10=2 m \omega+2 m^{\prime} \omega^{\prime}\right.$, where $\omega$ and $\omega^{\prime}$ are the two periods of the function, and $m, m^{\prime}=0, \pm 1, \pm 2$, . . . . $\pm \infty$ but 20 cannot, as we see, be $=0$, therefore $m$

and and $m^{\prime}$ cannot be simultaneously $=0$ )

$p u=-\frac{d^{2}}{d u^{2}} \log \sigma u$

form the proper basis for the theory of elliptic functions. $\sigma u$ is the one from which Weierstrass evolves the theory of elliptic functions, but, from the point of view of the elliptic integral, $p u$ is the one which, as we shall see, most naturally presents itself.

\section{II.}

Legendre (Traité des Fonctions Elliptiques, Vol. I. p. 35) showed that the integral $\mathrm{F}(k, \phi)$ was represented by the lemniscate

$$
\left(x^{2}+y^{2}\right)^{2}-a^{2}\left(x^{2}-y^{2}\right)=0
$$

in the particular case in which the modulus $k=\frac{1}{\sqrt{ } 2}$. 
The equation of the lemniscate is satisfied by the values

$$
\begin{aligned}
& x=a \cos \phi \sqrt{1-\frac{1}{2} \sin ^{2} \phi} \\
& y=\frac{a}{\sqrt{ } 2} \sin \phi \cos \phi
\end{aligned}
$$

But

$$
\begin{aligned}
d s^{2} & =d x^{2}+d y^{2} \\
& =\frac{a^{2} d \phi^{2}}{2\left(1-\frac{1}{2} \sin ^{2} \phi\right)} \\
\therefore s & =\frac{a}{\sqrt{ } 2} \int \frac{d \phi}{\sqrt{1-\frac{1}{2} \sin ^{2} \phi}}=\frac{a}{\sqrt{ } 2} \mathrm{~F}\left(\frac{1}{\sqrt{ } 2}, \phi\right),
\end{aligned}
$$

the arc $s$ being measured from the point $\phi=0$, i.e., from the extremity $(a, 0)$ of the axis of the lemniscate.

An important result follows from this, viz., from the addition, subtraction, multiplication, and division of elliptic functions it follows that arcs of a lemniscate can be added, subtracted, multiplied and divided algebraically just as the arcs of a circle can *.

After much time spent on the problem, Legendre (Traité des Fonctions Elliptiques, Vol. I. p. 36) invented a sextic curve which represented, for all values of $k$, the function $\mathrm{F}(k, \phi)$ with an algebraic function subtracted.

He took the curve whose coordinates satisfy the equations

$$
\left\{\begin{array}{l}
x=h \sin \phi\left(1+\frac{1}{3} m \sin ^{2} \phi\right) \dagger \\
y=k^{\prime} h \cos \phi\left(1+m-\frac{1}{3} m \cos ^{2} \phi\right)
\end{array}\right.
$$

where $k^{\prime}$ is the complementary modulus, i.e. $=\sqrt{1-k^{2}}$.

But $d s=\sqrt{d x^{2}+d y^{2}}=h \sqrt{1-k^{2} \sin ^{2} \phi}\left(1+m \sin ^{2} \phi\right) d \phi$

$\therefore(2) s=h \mathrm{E}(k, \phi)+\frac{m h}{3 k^{2}}\left[\left(2 k^{2}-1\right) \mathrm{E}(k, \phi)+k^{\prime 2} \mathrm{~F}(k, \phi)\right]$

$-k^{2} \sin \phi \cos \phi \sqrt{1-k^{2} \sin ^{2} \phi}$

- As early as 1716, long before Legendre had discovered the method of multiplying and dividing elliptic functions, Fagnano was able to multiply and divide ares of lemniscates (see Methodo per misurare la Lemniscata, pp. 343-368 of vol. 2 of his Produzioni Matematiche).

+ Cayley at $\$ 62$ of his Elliptic Functions states the value of $x$ and $y$ erroneously. 
In order that the second elliptic integral may disappear we must have

$$
m=\frac{3 k^{2}}{1-2 k^{2}}, \text { and therefore } h=\frac{3 k^{2}}{k^{\prime 2} m}=\frac{1-2 k^{2}}{k^{\prime 2}} .
$$

Hence we have

$$
s=\mathrm{F}(k, \phi)-\frac{k^{2}}{k^{2}} \sin \phi \cos \phi \sqrt{1-k^{2}} \overline{\sin ^{2} \phi} .
$$

The objection to this solution is that the arc $s$ represents not $F(k, \phi)$ but this integral minus an algebraic function. This algebraic quantity can be made to vanish by a suitable choice of the ends of the arc*, but it is not in general zero, and hence the sextic curve invented is not a perfect representation of the first elliptic transcendental. $\dagger$

The curve under consideration is, however, an interesting one. From (2) we see that $k^{2}$ may have any value provided it is not greater than $\frac{1}{2}$. On eliminating $\phi$ in (1) after having inserted the above values of $h$ and $m$, we find the equation of the curve to be

$$
y^{2}=k^{2}\left(2+x^{i}\right)^{2}\left(1-x^{3}\right)
$$

Hence the curve is of the sixth degree, and since there are no odd powers of $x$ and $y$ the curve is divided into four equal and similar parts by the axes of coordinates. The curve is of the form given in Fig. 11. On inserting the values of $h$ and $m,(1)$ gives

$$
\begin{aligned}
& x=\frac{\sin \phi}{k^{\prime 2}}\left(1-2 k^{2}+k^{2} \sin ^{2} \phi\right) \\
& y=\frac{\cos \phi}{k^{\prime}}\left(1+k^{2} \sin ^{2} \phi\right) .
\end{aligned}
$$

Hence, if $\quad \phi=0$ then $x=0, y=\frac{1}{k^{\prime}}$

$$
\text { if } \quad \phi=\frac{\pi}{2} \text { then } x=1, y=0
$$

* For example, if we integrate between the limits $\phi=0$ and $\phi=\frac{\pi}{2}$ then the additional algebraic function obviously vanishes, and we get $s_{1}=\mathrm{F}\left(k, \frac{\pi}{2}\right)$ where $s_{1}$ is the fourth part of the curve.

† Yet Legendre remarks (F. E., vol. ii., p. 591) " Le problème . . . . de trouver une courbe algébrique dont les arcs représentent généralement la fonction elliptique de première espèce $\mathrm{F}(k, \phi)$ parait $n$ ' admettre aucune autre solution." We shall see that we have travelled far since then. 
that is, $\mathrm{CA}$ is the semimajor axis of the curve and its length is $\frac{1}{k^{\prime}}$, and $C B$ is the semiminor axis and its length is 1 , and they lie respectively along the $y$ and the $x$ axes. The dotted curve outside is an ellipse on the same axes, and we see that the sextic curve differs very little from an ellipse.*

\section{III.}

Legendre, as we have seen, shored that, when the modulus is $\frac{1}{\sqrt{ } 2}, F(k, \phi)$ is represented by a - lemniscate. SERret (Liouville's Journal, vol. viii., p. 145) extended this by proving that, whatever the modulus, elliptic integrals of the first kind are represented by arcs of the cassinian oval, of which the lemniscate is only a particular case.

The equation of the cassinian in polar coordinates with centre as origin is

$$
r^{4}-2 a^{2} r^{2} \cos 2 \theta+a^{4}-b^{4}=0,
$$

$2 a$ being the distance between the two foci, and $b^{2}$ the product of the distances $d$ and $d^{\prime}$ of any point on the curve from the foci.

There are three cases accordingly as $b \bar{\equiv}$.

First case.

If $b=a$, then $d d^{\prime}=b^{2}=a^{2}$, and $d+d^{\prime}=2 a$, and (1) is the equation of the lemniscate studied by Legendre.

Second case.

If $b<a$, then $d d^{\prime}=b^{2}<a^{2}$, and $d+d^{\prime}<2 a$. Hence the curve consists of two loops equal to each other. (Fig. 12.)

- The case $k^{2}=1$ is generally solved, as we have seen, by the lemniscate which is only of the fourth degree, and its ares express the integral $F(k, \phi)$ without any additional algebraical quantity. If we take the solution of this case given by (3) we find that the equation is

$$
y^{2}=2-\frac{1}{2} x^{2}-\frac{3}{2} x^{\frac{4}{3}}
$$

Athough this curve is not so simple as the lemniscate it has the advantage of differing little from an ellipse. 
Put $\frac{b^{2}}{a^{2}}=\sin 2 \phi$, so that $2 \phi$ is the angle between the tangents drawn from the centre.*

If $p$ be the length of the perpendicular from the centre on a tangent, and $r$ the radius vector to the point of contact, then from (1) it can be shown that the $p, r$ equation of the cassinian with centre as origin is

$$
p=\frac{r^{4}+b^{4}-a^{4}}{2 b^{2} r}
$$

But for the rectification of any curve we have

Hence in this case

$$
s=\int \frac{r^{2} d \theta}{p} .
$$

$$
s=b^{2} \int \frac{2 r^{3} d \theta}{r^{4}+b^{4}-a^{4}}
$$

Solving (1), after substituting $a^{4} \sin ^{2} 2 \phi$ for $b^{4}$, we get

$$
r=a\left(\cos 2 \theta \pm \sqrt{\cos ^{2} 2 \theta-\cos ^{2} 2 \phi}\right)^{\frac{1}{2}}
$$

$\therefore$ (2) gives $s=b^{2} \int \frac{2 a^{3}\left(\cos 2 \theta \pm \sqrt{\cos ^{2} 2 \theta-\cos ^{2} 2 \phi}\right)^{\frac{3}{2}} d \theta}{a^{4}\left(\cos 2 \theta \pm \sqrt{\cos ^{2} 2 \theta-\cos ^{2} 2 \phi}\right)^{2}+b^{4}-a^{4}}$

Substituting for $b^{4}$ and simplifying we get

$$
s=\frac{b^{2}}{a} \int \frac{\left(\cos 2 \theta \pm \sqrt{\cos ^{2} 2 \theta-\cos ^{2} 2 \phi}\right)^{\frac{1}{2}} d \theta}{\sqrt{\cos ^{2} 2 \theta-\cos ^{2} 2 \phi}}
$$

If we integrate between $\theta_{0}$ and $\theta$, we see that owing to the double sign in the numerator the radius vectors corresponding to these initial angles will determine upon the curve two ares which we may represent by $s\left(\theta_{0}, \theta\right)$ and $\sigma\left(\theta_{0}, \theta\right)$, or by $s(\theta)$ and $\sigma(\theta)$ if we integrate between 0 and $\theta$.

* This is perhaps most easily seen by noticing that the condition for tangency is that (1) have equal roots, that is $a^{4} \cos ^{2} 2 \theta=a^{4}-b^{4}$, where $2 \theta$ is the angle between the tangents. Hence

$$
\begin{aligned}
b^{4} & =a^{4}\left(1-\cos ^{2} 2 \theta\right) \\
& =a^{4} \sin ^{2} 2 \theta
\end{aligned}
$$

$\therefore \frac{b^{2}}{a^{2}}=\sin 2 \theta$. But by hypothesis $\frac{b^{2}}{a^{2}}=\sin 2 \phi$. Therefore $2 \phi=2 \theta$, i.e., $2 \phi$ is equal to the angle between the tangents drawn from the centre. 
Hence from (3) we have

(4) $\mathrm{s}\left(\theta_{0}, \theta\right)=\frac{b^{2}}{a} \int_{\theta_{0}}^{\theta} \frac{\left(\cos 2 \theta+\sqrt{\cos ^{2} 2 \theta-\cos ^{2} 2 \phi}\right)^{\frac{1}{2}} d \theta}{\sqrt{\cos ^{2} 2 \theta-\cos ^{2} 2 \phi}}$

(5) $\sigma\left(\theta_{0}, \theta\right)=\frac{b^{2}}{a} \int_{\theta_{0}}^{\theta} \frac{\left(\cos 2 \theta-\sqrt{\cos ^{2} 2 \theta-\cos ^{2} 2 \phi}\right)^{\frac{1}{2}} d \theta}{\sqrt{\cos ^{2} 2 \theta-\cos ^{2} 2 \phi}}$

Whence we get

(6) $s\left(\theta_{0}, \theta\right)+\sigma\left(\theta_{0}, \theta\right)=\frac{2 t b^{2}}{a} \int_{\theta_{0}}^{\theta} \frac{d \theta}{\sqrt{\cos 2 \theta-\cos 2 \phi}}$

(7) $s\left(\theta_{0}, \theta\right)-\sigma\left(\theta_{0}, 0\right)=\frac{2 \pm b^{2}}{a} \int_{\theta_{0}}^{\theta} \frac{d \theta}{\sqrt{\cos 2 \bar{\theta}+\cos 2 \phi}}$

If in (6) we put

$$
\sin \theta=\sin \phi \sin \chi
$$

and in (7) put

$$
\sin \theta=\cos \phi \sin \psi
$$

then from (6) and (7) respectively we obtain

(10) $s\left(\theta_{0}, \theta\right)+\sigma\left(\theta_{0}, \theta\right)=\frac{b^{2}}{a} \int_{\chi_{0}}^{\chi} \frac{d \chi}{\sqrt{1-\sin ^{2} \phi \sin ^{2} \chi}}$

(11) $s\left(\theta_{0}, \theta\right)-\sigma\left(\theta_{0}, \theta\right)=\frac{b^{2}}{a} \int_{\psi / \psi_{0}}^{\psi} \frac{d \psi}{\sqrt{1-\cos ^{2} \phi \sin ^{2} \psi}}$

If we make $\theta_{0}=0$, we see from the relations (8) and (9) that

$$
\chi_{0}=0 \text { and } \psi_{0}=0
$$

$\therefore(10)$ and (11) become respectively

(12) $\frac{a}{b^{2}}[s(\theta)+\sigma(\theta)]=\int_{0}^{\chi} \frac{d \chi}{\sqrt{1-\sin ^{2} \phi \sin ^{2} \chi}}=\mathrm{F}(\sin \phi, \chi)$

(13) $\frac{a}{b^{2}}[s(\theta)-\sigma(\theta)]=\int_{0}^{\psi} \frac{d \psi}{\sqrt{1-\cos ^{2} \phi \sin ^{2} \psi}}=\mathbf{F}(\cos \phi, \psi)$ 
But $\sin 2 \phi=\frac{b^{2}}{a^{2}}$, whence it can easily be shown that

$$
\begin{aligned}
& \sin \phi=\frac{1}{2} \sqrt{1+\frac{b^{2}}{a^{2}}}-\frac{1}{2} \sqrt{1-\frac{b^{2}}{a^{2}}} \\
& \cos \phi=\frac{1}{2} \sqrt{1+\frac{b^{2}}{a^{2}}}+\frac{1}{2} \sqrt{1-\frac{b^{2}}{a^{2}}}
\end{aligned}
$$

Hence the moduli, $\sin \phi$ and $\cos \phi$, of the elliptic integrals on the right hand of (12) and (13) are complementary. *

\section{Third case.}

If $b>a$, then $d d^{\prime}=b^{2}>a^{2}$, and $d+d^{\prime}>2 a$, and the cassinian takes the form shown in Figure 13.

This case might be discussed in exactly the same way as the foregoing; we need however only state the results.

In this instance we put $\frac{a^{2}}{b^{2}}=\sin 2 \phi$

We then get two equations corresponding to (6) and (7), viz.,

$$
\begin{aligned}
& s\left(\theta_{0}, \theta\right)+\sigma\left(\theta_{0}, \theta\right)=\frac{2 t b^{2}}{a} \int_{\theta_{0}}^{\theta} \frac{\left(\operatorname{cotan} 2 \phi+\sqrt{\cos ^{2} 2 \theta+\operatorname{cotan}^{2} 2 \phi}\right)^{t}}{\sqrt{\cos ^{2} 2 \theta+\operatorname{cotan}^{2} 2 \phi}} d \theta \\
& s\left(\theta_{0}, \theta\right)-\sigma\left(\theta_{0}, \theta\right)=\frac{2 \downarrow b^{2}}{a} \int_{\theta_{0}}^{\theta} \frac{\left(-\operatorname{cotan} 2 \phi+\sqrt{\cos ^{2} 2 \theta+\operatorname{cotan}^{2} 2 \phi}\right)^{t}}{\sqrt{\cos ^{2} 2 \theta+\operatorname{cotan}^{2} 2 \phi}} d \theta
\end{aligned}
$$

In this case the relations connecting $\theta, \chi$, and $\psi$ corresponding to (8) and (9) are

$$
\begin{aligned}
& \sqrt{\cos ^{2} 2 \theta+\operatorname{cotan}^{2} 2 \phi}=\frac{1-2 \sin ^{2} \phi \sin ^{2} \chi}{\sin 2 \phi} \\
& \sqrt{\cos ^{2} 2 \theta+\operatorname{cotan}^{2} 2 \phi}=\frac{1-2 \cos ^{2} \phi \sin ^{2} \psi}{\sin 2 \phi}
\end{aligned}
$$

- Of course all this applies equally to the case $b=a$, for then $\phi=\frac{\pi}{4}$, in which case the two loops meet at the centre and we get the lemniscate, and from (5) we that the aro represented by $\sigma\left(\theta_{0}, \theta\right)$ disappears as it ought to do since the polar equation of the lemniscate is only of the second degree in $r$. 
Then the results corresponding to (12) and (13) are

$$
\begin{aligned}
& \frac{1}{b}[s(\theta)+\sigma(\theta)]=F(\sin \phi, \chi) \\
& \frac{1}{b}[s(\theta)-\sigma(\theta)]=F(\cos \phi, \psi)
\end{aligned}
$$

where the moduli $\sin \phi, \cos \phi$ are again complementary, viz.,

$$
\begin{aligned}
& \sin \phi=\frac{1}{2} \sqrt{1+\frac{a^{2}}{b^{2}}}-\frac{1}{2} \sqrt{1-\frac{a^{2}}{b^{2}}} \\
& \cos \phi=\frac{1}{2} \sqrt{1+\frac{a^{2}}{b^{2}}}+\frac{1}{2} \sqrt{1-\frac{a^{2}}{b^{2}}}
\end{aligned}
$$

From (12), (13), (14), and (15) we see that in every case and whatever the modulus, the elliptic integral of the first kind can be represented by the sum or difference of two arcs of a cassinian oval, and conversely (by addition and substraction of (12) and (13), or of (14) and (15)) the arc of the cassinian oval is represented by the sum or difference of two elliptic integrals of the first kind whose moduli are complementary.*

IV.

The next to attack the problem we are investigating was $W$. RoBrets of Dublin (Liouville's Journal, vol. viii,, p. 263, vol. ix., p. 155, vol. x., p. 297).

- Comparing Serret's result with Legendre's in the last section, it should be observed that if in (8) we make $\theta-\phi$, then $x=\frac{\pi}{2}$, hence, the amplitude being a right angle, we may write $s(\theta)+\sigma(\theta)=s_{1}$ where $s_{2}$ is as before onefourth of the total length of the curve. Then from (12) we get

$$
\frac{a}{b^{2}} s_{1}=\mathrm{F}\left(\sin \phi, \frac{\pi}{2}\right)
$$

Similarly, if in (13a) we put $\theta=\frac{\pi}{4}$ (and of course $\left.\sin 2 \phi=\frac{a^{2}}{b^{2}}\right)$ then $\chi=\frac{\pi}{2}$, and we then above get from (14)

$$
\frac{8_{1}}{b}=\mathrm{F}\left(\sin \phi, \frac{\pi}{2}\right)
$$

From these two equation we see that, just as in Legendre's solution, by properly choosing the ends of the arc, i.e., by integrating between suitsble limits, the elliptio integral of the first kind for any modulus and without any extra algebraical quantity is represented by the arc of a cassinian oval. 
He showed that the curves in which a sphere is cut by a cone of the second order, one of whose principal external axes is a diameter of the sphere, can be rectified by means of an expression containing all three kinds of elliptic integrals. Taking the particular case in which the elliptic integrals of the second and third kinds vanished he got a single curve on the surface of the sphere, whose arc reckoned from the extremity of the semi-axis was expressed by an elliptic integral of the first kind. He showed that the form of this curve was similar to that of the lemniscate, and that it was the locus of points on the sphere the product of whose distances from two fixed points on the sphere was constant.* Hence Kiepert called this curve, discovered by Roberts in 1843 , the "spherical lemniscate."

V.

SerRet returned to the problem of the geometrical representation of the first elliptic integral, and wrote an important Memoir on the subject (Liouville's Journal, vol. x., p. 257) and an Additional Note (p. 286). A new method of solution was suggested to him by the fact that the equation of the lemniscate

is satisfied by

$$
\left(x^{2}+y^{2}\right)^{2}-a^{2}\left(x^{2}-y^{2}\right)=0
$$

$$
x=a \frac{z+z^{3}}{1+z^{4}}, \quad y=a \frac{z-z^{3}}{1+z^{4}}
$$

whence (1)

$$
d s=\sqrt{d x^{3}+d y^{2}}=\frac{a \sqrt{2} d z}{\sqrt{1+z^{4}}}
$$

so that the arc is expressible as an elliptic integral. +

* If the radius of the sphere were made infinitely great it would become a plane, and the curve on it would become a plane lemniscate.

† This leads to exactly the same solution as Legendre's on p. 7, for let

$$
\begin{aligned}
& \sin \phi=\frac{1-z^{2}}{\sqrt{1+z^{4}}} \text {, and therefore } \cos \phi=\frac{z \sqrt{ } 2}{\sqrt{1+z^{4}}} \\
& \quad \text { and } \sqrt{1-\frac{1}{2} \sin ^{2} \phi}=\frac{1}{\sqrt{ } 2} \frac{1+z^{2}}{\sqrt{1+z^{4}}}
\end{aligned}
$$

Whence, after differentiating and reducing, we get

$$
\frac{d \phi}{\sqrt{1-\frac{1}{2} \sin ^{2} \phi}}=\frac{2 d z}{\sqrt{1+z^{4}}}
$$

But

$$
s=a \sqrt{2} \int \frac{d z}{\sqrt{1+z^{4}}}=\frac{a}{\sqrt{2}} \int \frac{d \phi}{\sqrt{1-\frac{1}{2} \sin ^{2} \phi}}=\frac{a}{\sqrt{ } 2} \mathrm{~F}\left(\frac{1}{\sqrt{ } 2}, \phi\right)
$$


By generalising this method he was able to prove that not merely the lemniscate and the cassinian but an infinite number of plane curves represented the first elliptic transcendental.

In the generalisation of equation (1) he discusses

$$
d s^{2}=d x^{2}+d y^{2}=\mathrm{C}^{2} \frac{d z^{2}}{\mathrm{P}}
$$

where $\mathrm{P}$ is a quartic rational function of $z$, and $\mathrm{C}$ a constant.

Of course $\mathbf{P}$ does not include multiple factors else, after extracting the square root of each side, $(1 a)$ would not lead to an elliptic integral.

Nor can $\mathbf{P}$ have real factors, for since

$$
d x+i d y \quad \cdot \quad d x-i d y=\mathrm{C}^{2} \frac{d z^{2}}{\overline{\mathrm{P}}}
$$

we see that every real value of $z$ which would make the right-hand side of (2) infinite would necessarily make both $d x+i d y$ and $d x-i d y$ infinite, and this is impossible since $P$ has not multiple factors.

Therefore $\mathbf{P}$ can only have four imaginary factors, and, since their product is real, they must be conjugate two and two, say $b$ and $\beta$ conjugate and $c$ and $\gamma$ conjugate. Then ( $1 a)$ becomes

$$
d s^{2}=d x^{2}+d y^{2}=\frac{\mathrm{C}^{2} d z^{2}}{(z-b)(z-\beta)(z-c)(z-\gamma)}
$$

We may therefore let $\mathrm{P}=p . \pi$, where $p$ and $\pi$ are two conjugate functions each of the second degree in $z$. Therefore (2) gives

$$
\frac{d x+i d y}{\mathrm{o} \frac{d z}{p}} \cdot \frac{d x-i d y}{\mathrm{c} \frac{d z}{\pi}}=1
$$

The two functions on the left hand side are, therefore, conjugate and have 1 for modulus. Serret goes on to prove in section II. of his Memoir that these conjugate quantities must have the form

or (5)

$$
\frac{d x+i d y}{\mathrm{C} \frac{d z}{p}}=\frac{\Delta r^{2}}{\mathrm{D} \rho^{2}} \text { and } \frac{d x-i d y}{\mathrm{C} \frac{d z}{\pi}}=\frac{\mathrm{D} \rho^{2}}{\Delta r^{2}}
$$

$$
x+i y=\mathrm{O} \int \frac{\Delta r^{2} d z}{p \cdot \mathrm{D} \rho^{2}} \text { and } x-i y=\mathrm{C} \int \frac{\mathrm{D} \rho^{2} d z}{\pi \cdot \Delta r^{2}}
$$


where $r$ is any integral function of the variable $z$, D the G.C.M. of $r$ and its first derivative, and $\rho$ and $\Delta$ the conjugate complex quantities of $r$ and $D$ respectively.

But by a real and rational substitution

$\frac{d z^{2}}{(z-b)(z-\beta)(z-c)(z-\gamma)}$ may be transformed into $\frac{d z_{1}{ }^{2}}{\left(z_{1}^{2}-a^{2}\right)\left(z_{1}^{2}-a^{2}\right)}$ where $a$ and $\alpha$ are conjugate complex quantities.

Hence we may write (3) as follows

and $\therefore$

$$
d z^{2}=d x^{2}+d y^{2}=\frac{\mathbf{C}^{2} d z^{2}}{\left(z^{2}-a^{2}\right)\left(z^{2}-a^{2}\right)}
$$

$$
p=z^{2}-a^{2}, \quad \pi=z^{2}-a^{2} .
$$

For the success of Serret's method it was necessary to choose $r$ and $\rho$ so that they contained no factors except those of $p$ and $\pi$ respectively. Wherefore he put

$$
\begin{cases}r=(z-a)^{m}(z+a)^{n}, & \mathrm{D}=(z-a)^{m-1}(z+a)^{n-1} \\ \rho=(z-a)^{m}(z+a)^{n}, & \Delta=(z-a)^{m-1}(z+a)^{n-1} .\end{cases}
$$

Therefore from (5)

$$
x+i y=\mathrm{C} \int \frac{(z-x)^{m}(z+a)^{n} d z}{(z-a)^{m+1}(z+a)^{n+1}} \quad=\mathrm{C} \int f(z) d z, \text { sagy }
$$

For shortness let

$$
\left\{\begin{array}{l}
\phi(z)=f(z)(z-a)^{m+1}=\frac{(z-a)^{m}(z+a)^{n}}{(z+a)^{n+1}} \\
\psi(z)=f(z)(z+a)^{n+1}=\frac{(z-a)^{m}(z+a)^{n}}{(z-a)^{m+1}}
\end{array}\right.
$$

Now

$$
f(z)=\frac{(z-a)^{m}(z+a)^{n}}{(z-a)^{m+1}(z+a)^{n+1}}
$$

Therefore by partial fractions

$$
\begin{aligned}
& f(z)=\frac{\phi(a)}{(z-\alpha)^{m+1}}+\frac{\phi^{\prime}(\alpha)}{1 !(z-a)^{m}}+\ldots . . .++\frac{\phi^{(m)}(\alpha)}{m !(z-\alpha)} \\
& +\frac{\psi(-a)}{(z+a)^{n+1}}+\frac{\psi^{\prime}(-a)}{1 !(z+a)^{n}}+\ldots . . . \quad+\frac{\psi^{(n)}(-a)}{n !(z+a)}
\end{aligned}
$$

We wish to find the condition that the curve determined by $x$ and $y$ in (8) be algebraic. 
Since $x+i y=\mathrm{C} \int f(z) d z$ we see from (10) that in order that $x$ and $y$ be purely algebraic and not contain a logarithmic part the conditions are

$$
\phi^{(m)}(\alpha)=0 \text { and } \psi^{(n)}(-\alpha)=0 .
$$

But from (10) by multiplying up we get

$$
\begin{aligned}
& f(z)(z-a)^{m+1}(z-a)^{n+1} \text { or }(z-a)^{m}(z+a)^{n} \\
& =(z+a)^{n+1}\left\{\phi(a)+\frac{\phi^{\prime}(a)}{1 !}(z-a)+\cdot .+\frac{\phi^{(m)}(a)}{m !}(z-a)^{m}\right\} \\
& +(z-a)^{m+1}\left\{\psi(-a)+\frac{\psi^{\prime}(-a)}{1 !}(z+a)+. \cdot+\frac{\psi^{(n)}(-a)}{n !}(z+a)^{n}\right\}
\end{aligned}
$$

Arranging the right-hand side according to powers of $\approx$ we get

$$
(z-a)^{m}(z+a)^{n}=\left\{\frac{\phi^{(m)}(a)}{m !}+\frac{\psi^{(n)}(-\alpha)}{n !}\right\} z^{m+n+1}+\ldots .
$$

wherefore we see that

$$
\frac{\phi^{(m)}(a)}{m !}+\frac{\psi^{(n)}(-a)}{n !}=0
$$

which shows that if one of the conditions in (11) be satisfied the other will necessarily follow.

Hence the sufficient and necessary condition that the curves determined by $x$ and $y$ in

$$
x+i y=\mathrm{C} \int \frac{(z-a)^{m}(z+a)^{n} d z}{(z-a)^{m+1}(z+a)^{n+1}}
$$

be algebraic is $\phi^{(m)}(a)=0$. And the arcs of those curves are elliptic integrals of the first kind, for

$$
\begin{aligned}
& d x+i d y=\mathrm{C} \frac{(z-a)^{m}(z+a)^{n} d z}{(z-a)^{m+1}(z+a)^{n+1}} \\
& \therefore \text { also } \quad d x-i d y=\mathrm{C} \frac{(z-a)^{m}(z+a)^{n} d z}{(z-a)^{m+1}(z+a)^{n+1}}
\end{aligned}
$$


Wherefore, by multiplication,

or

$$
\begin{gathered}
d s^{2}=d x^{2}+d y^{2}=\frac{\mathrm{C}^{2} d z^{2}}{\left(z^{2}-a^{2}\right)\left(z^{2}-a^{3}\right)} \\
s=\mathrm{C} \int \frac{d z}{\sqrt{\left(z^{2}-a^{2}\right)\left(z^{2}-a^{2}\right)}},
\end{gathered}
$$

which is an elliptic integral of the first kind.

Also, since $m$ and $n$ may be anything whatever, we have a double infinity of curves, analogous to the lemniscate, represented by (8), and since the arcs of those curves are elliptic integrals of the first kind they can be added, subtracted, multiplied, and divided just as the arcs of the circle and lemniscate can.

After performing the integration in (8) and substituting the values of $a$ and $\alpha$ that satisfy the conditions, we equate the real and imaginary parts on each side and so obtain the coordinates $x$ and $y$ of the required curve as functions of the parameter $z$.

We shall take two examples to illustrate this infinite group of curves discovered by Serret.

\section{First Example.}

If $m=1, n$ being any integer, then the condition that the curve be algebraic is $\phi^{\prime}(a)=0$.

Now, from (9) we see that

$$
\phi(z)=\frac{(z-a)(z+a)^{n}}{(z+a)^{n+1}}
$$

whence, obtaining $\phi^{\prime}(z)$ and putting $a$ instead of $z$ and equating to zero, we get

$$
\frac{a^{2}+a^{2}}{a a}=2 \frac{n-1}{n+1}
$$

But, if we reduce

$$
\mathrm{C} \int \frac{d z}{\sqrt{\left(z^{2}-a^{2}\right)\left(z^{2}-a^{2}\right)}} \text { to the standard form } 0 \int \frac{d \phi}{\sqrt{1-k^{2} \sin ^{2} \phi}}
$$

we find that $k^{2}$, the square of the modulus, is $=\frac{(a+a)^{2}}{4 a \alpha}$ 
wherefore, from (13)

$$
k^{2}=\frac{(a+a)^{2}}{4 a \alpha}=\frac{n}{n+1}
$$

Hence if we take $a$ and its conjugate $a$ so that they satisfy (13), then the curves defined by

$$
x+i y=\mathrm{C} \int \frac{(z-a)(z+a)^{n} d z}{(z-\alpha)^{2}(z+\alpha)^{n+1}}
$$

will be algebraic, and their arcs will be represented by the integral $\mathrm{C} \int \frac{d z}{\sqrt{\left(z^{2}-a^{2}\right)\left(z^{2}-a^{2}\right)}}$ whose modulus $k$ is $=\sqrt{\frac{n}{n+1}}$; and, as $n$ may be any integer whatever, we have an infinite number of such curves.*

\section{Second Example.}

Next take $m=2$. Then the condition that the curves be algebraic is $\phi^{\prime \prime}(a)=0$.

But $\phi(z)=\frac{(z-a)^{2}(z+a)^{n}}{(z+a)^{n+1}}$. Differentiating twice and putting $a$ for $z, \phi^{\prime \prime}(a)=0$ gives

$$
\frac{a^{2}+a^{2}}{a \alpha}=\frac{2(n+1)(n-2) \pm 4 \sqrt{2 n(n+1)}}{(n+1)(n+2)} .
$$

If $a$ and $a$ satisfy this condition, then the arcs of the algebraic curves defined by

$$
x+i y=\mathrm{C} \int \frac{(z-a)^{\prime \prime}(z+a)^{n} d z}{(z-\alpha)^{n}(z+a)^{n+1}}
$$

will be represented by the elliptic integral $\mathrm{C} \int \frac{d z}{\sqrt{\left(z^{2}-a^{2}\right)\left(z^{2}-a^{2}\right)}}$,

* If we make $n=1$ then $k=\frac{1}{\sqrt{ } 2}$, i.e., the lemniscate is the simplest case of this infinite class of curves. To obtain its equation we may proceed thus :If $n=1$ then from (13) $a^{2}+a^{2}=0$ and $a \alpha=1$, whence $a^{2}=i$ and $a^{2}=-i$. Putting these values in (14) we get, after one or two steps,

$$
x+i y=\mathrm{C} \frac{z^{3}+i z}{z^{4}+1}
$$

whence $x=\mathrm{C} \frac{z^{3}}{z^{4}+1}, y=\mathrm{C} \frac{z}{z^{4}+1}$. Eliminating $z$ between these, we get $\left(x^{2}+y^{2}\right)^{2}=C^{2} x y$. 
the square of whose modulus is from (15)

$$
k^{2}=\frac{(a+a)^{2}}{4 a a}=\frac{n(n+1) \pm \sqrt{2 n(n+1)}}{(n+1)(n+2)} .
$$

If we also make $n=2$, then (15) gives

$$
a^{2}+a^{2}=\frac{2}{\sqrt{3}}, \text { and } a a=1
$$

whence

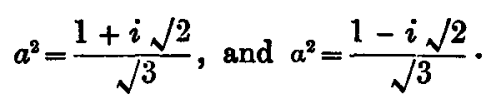

Putting these values in (16) we get ultimately

$$
x+i y=0 \frac{z^{3}-\frac{5-i \sqrt{ } 2}{3 \sqrt{ }} z}{\left(z^{2}-\frac{1-i \sqrt{ } 2}{\sqrt{3}^{3}}\right)^{2}}
$$

On rationalising the denominator and then equating the real and imaginary parts on each side we obtain for the coordinates

$$
\begin{aligned}
& x=\mathrm{C} \frac{z^{z}-\frac{11 z^{5}}{3 \sqrt{ } 3}+\frac{11 z^{3}}{9}+\frac{z}{9 \sqrt{ } 3}}{\left(z^{4}-\frac{2}{\sqrt{ } 3} z^{2}+1\right)^{2}} . \\
& y=C \frac{\frac{5 \sqrt{ } 2}{3} z^{5}-\frac{14 \sqrt{ } 2}{9} z^{3}+\frac{11 \sqrt{ } 2 z}{9 \sqrt{ } 3}}{\left(z^{4}-\frac{2}{\sqrt{ } 3} z^{3}+1\right)^{2}}
\end{aligned}
$$

The curve is of the sixth degree, and, in an Additional Note to his Memoir, Serret shows that its equation in polar coordinates is

$$
9 r^{2}\left(r^{2}-2 \mathrm{C}^{2} \cos 2 \theta\right)^{2}+8 \mathrm{C}^{4}\left(r^{2}-2 \mathrm{C}^{2} \cos 2 \theta\right)+\frac{16 \mathrm{C}^{6}}{9 \sqrt{ } 3}=0
$$


where $2 \mathrm{C}$ ( $c f$. equations $(1)$ and $\left.\left(1^{\alpha}\right)\right)$ is the distance between the two foci of the curve.*

VI.

As we have said on p. 16, Serret could only satisfy the conditions that the curve be algebraic if $r$ contained no other factors than those of $p$, viz., $z-a$ and $z+a$. He said, "En général pour une forme déterminée des polynômes $r$ et $\rho$ les conditions ne pouront subsister en même temps; mais il existe un cas très étendu, où elles pourront toujours être satisfaites, c'est celui, où les polynômes $r$ et $\rho$ ne renfermeront que les facteurs linéaires des polynómes données $p$ et $\pi . "$

L. KIEPRRT in a dissertation entitled De curvis quarum arcus integralibus ellipticis primi generis exprimuntur (Berlin, 1870) showed that this restriction was not necessary, and that therefore

* We have always taken the condition $\phi^{(m)}(\alpha)=0$, but of course we take the one or the other of the conditions (11) according as $m$ or $n$ is the smaller. If the smaller exceeds 2 then the condition for $a$ and $a$ will be at least of the third degree and cannot in general be resolved, but this does not affect the reasoning by which we obtained an infinity of curves for any given value of the smaller of $m$ or $n$.

Moreover, $n$ has throughout been supposed integral, but M. Liouville proved in Liouville's Journal, vol. x. p. 293, that $n$ need not be integral but only rational in order that Serret's infinity of curves for $x$ and $y$ remain algebraic.

We have seen that $\phi^{(m)}(a)=0$ leads always to a relation symmetrical and homogeneous in $a$ and $a$. Serret shows that this relation in its most general form is

$$
\begin{array}{r}
\sum_{p=0}^{m} \sum_{q=0}^{n}(-1)^{p+q} \frac{(n+m-p+1) !(n+m-q+1) !}{(m-p+1) !(m-q+1) !(p+1) !(q+1) !(n+m-p-q+1) !} \\
\times(2 a)^{p}(2 a)^{q}(a+a)^{2 m-p-q}=0
\end{array}
$$

CaYLeY (Elliptic Functions, chap. xv.) states the relation between $a$ and $a$ much more briefly thus :-Putting $\xi$ for $\frac{(a+a)^{2}}{4 a a}$, the square of the modulus, then the relation is

$$
\frac{1}{\xi^{n-m}}\left(\frac{d}{d \xi}\right)^{m} \xi^{n}(\xi-1)^{m}=0
$$

If we make $m=1$ or 2 , etc., then this gives the same values of the modulus as we have obtained above. 
Serret's class of curves could be greatly extended. For let $b_{1}, b_{2}$, . . $b_{x}$ be other $x$ imaginaries, and $\beta_{1}, \beta_{2}, \ldots . \beta_{x}$ their conjugates. Also let

$$
r=(z-a)^{m n}(z+a)^{n}\left(z-b_{1}\right)^{n_{1}}\left(z-b_{2}\right)^{n_{2}} \text {. . . . }\left(z-b_{x}\right)^{n_{x}}
$$

Then as in V. (7) we will get corresponding equations for $\rho$, $\mathrm{D}$ and $\Delta$. Also as before let $p=z^{2}-a^{2}, \pi=z^{2}-a^{2}$.

Then corresponding to $\mathrm{V}$. (8) we get

$$
\begin{aligned}
& \text { (1) } x+i y=\int \frac{\Delta y^{2} d z}{p \cdot \bar{D} \rho^{2}} \\
= & \int \frac{(z-a)^{m}(z+a)^{n}\left(z-b_{1}\right)^{n_{1}+1}\left(z-b_{2}\right)^{n_{2}+1} \ldots \ldots \ldots\left(z-b_{x}\right)^{n_{x}+1} d z}{(z-a)^{m+1}(z+a)^{n+1}\left(z-\beta_{1}\right)^{n_{1}+1}\left(z-\beta_{2}\right)^{n_{2}+1} \ldots\left(z-\beta_{x}\right)^{n_{x}+1}} \\
= & \int f(z) d z, \text { say. }
\end{aligned}
$$

Also V. (9) now becomes

$$
\left\{\begin{array}{l}
\phi(z)=f(z)(z-a)^{n+1} \\
\psi(z)=f(z)(z+\alpha)^{n+1} \\
\chi_{1}(z)=f(z)\left(z-\beta_{1}\right)^{n_{1}+1} \\
\cdot \cdot \cdot \cdot \cdot \cdot \cdot \\
\chi_{x}(z)=f(z)\left(z-\beta_{x}\right)^{n_{x}+1}
\end{array}\right.
$$

Instead of V. (10) we have

$$
\begin{aligned}
f(z) & =\frac{\phi(a)}{(z-a)^{m+1}}+\frac{\phi^{\prime}(\alpha)}{1 !(z-a)^{m}}+\ldots+\frac{\phi^{(m)}(a)}{m !(z-a)} \\
& +\frac{\psi(-a)}{(z+a)^{n+1}}+\frac{\psi^{\prime}(-a)}{1 !(z+\alpha)^{n}}+\ldots+\frac{\psi^{(n)}(-a)}{n !(z+\alpha)} \\
& +\frac{\chi_{1}\left(\beta_{1}\right)}{\left(z-\beta_{1}\right)^{n+1}}+\frac{\chi_{1}^{\prime}\left(\beta_{1}\right)}{1 !\left(z-\beta_{1}\right)_{1}}+\ldots+\frac{\chi_{1}^{(n)}\left(\beta_{1}\right)}{n_{1} !\left(z-\beta_{1}\right)} \\
& +\cdot \cdot \cdot \cdot \cdot \cdot \cdot \cdot \frac{\chi_{x}^{\left(n_{x}\right)}\left(\beta_{x}\right)}{n_{x} !\left(z-\beta_{x}\right)} .
\end{aligned}
$$


On multiplying up and comparing the powers of $z$ on the left and right-hand side of this we get as in V. (12)

(3) $\frac{\phi^{(m)}(\alpha)}{m !}+\frac{\psi^{(n)}(-a)}{n !}+\frac{\chi_{1}{ }^{\left(n_{1}\right)}\left(\beta_{1}\right)}{n_{1} !}+\ldots+\frac{\chi_{x}{ }^{\left(n_{x}\right)}\left(\beta_{x}\right)}{n_{x} !}=0$

In order that $x$ and $y$ be purely algebraic we get $x+2$ conditions corresponding to V. (11), viz.,

$\phi^{(m)}(\alpha)=0, \quad \psi^{(n)}(-\alpha)=0, \quad \chi_{1}{ }^{\left(n_{1}\right)}\left(\beta_{1}\right)=0 \ldots \chi_{x}{ }^{\left(n_{x}\right)}\left(\beta_{x}\right)=0$.

But because of (3) these reduce to $x+1$ conditions, which can always be fulfilled for we have $x+1$ quantities at our disposal, viz., or their conjugates $a, \beta_{1}, \beta_{2}$, . . . . $\beta_{x}$.

$$
a, b_{1}, b_{2}, \cdot . \cdot b_{x}
$$

Thus the conditions that the curves represented by (1) be algebraic can be fulfilled, and since $p$ and $\pi$ are, as we have said, the same as before, the arcs of these curves will be represented as formerly by an elliptic integral of the first kind, viz.,

$$
s=\int \frac{d z}{\sqrt{\left(z^{2}-a^{2}\right)\left(z^{2}-a^{2}\right)}} .
$$

\section{EXAMPLE.}

Kiepert does not give a direct example of (1) we shall therefore fully discuss the following case.

Let

$\therefore$ from (1)

$$
r=(z-a)(z+a)(z-b)^{n}
$$

$$
x+i y=\int \frac{(z-a)(z+a)(z-b)^{n+1}}{(z-a)^{2}(z+a)^{2}(z-\beta)^{n+1}} d z
$$

and from (2) we get

$$
\begin{aligned}
& \phi(z)=\frac{(z-a)(z+a)(z-b)^{n+1}}{(z+a)^{2}(z-\beta)^{n+1}} \\
& \psi(z)=\frac{(z-a)(z+a)(z-b)^{n+1}}{(z-a)^{2}(z-\beta)^{n+1}} \\
& \chi(z)=\frac{(z-a)(z+a)(z-b)^{n+1}}{(z-a)^{2}(z+a)^{2}}
\end{aligned}
$$


That the curves $(4)$ be algebraic the conditions are

$$
\begin{aligned}
& \phi^{\prime}(a)=0, \quad \psi^{\prime}(-a)=0 . \\
& \text { Now } \frac{\phi^{\prime}(z)}{\phi(z)}=\frac{1}{z-a}+\frac{1}{z+a}-\frac{2}{z+a}+(n+1)\left\{\frac{1}{z-b}-\frac{1}{z-\beta}\right\} \\
& =\frac{2 z a+2 a^{2}}{\left(z^{2}-a^{2}\right)(z+a)}+\frac{(n+1)(b-\beta)}{(z-b)(z-\beta)} .
\end{aligned}
$$

Hence the condition $\phi^{\prime}(\alpha)=0$ evidently gives

(5) $\left(a^{2}+a^{2}\right)\left\{a^{2}-a(b+\beta)+b \beta\right\}+(n+1) \alpha\left(\alpha^{2}-a^{2}\right)(b-\beta)=0$.

The second condition, $\psi^{\prime}(-\alpha)=0$, will obviously be got from this by putting $-\alpha$ instead of $\alpha$, therefore

(6) $\left(a^{2}+a^{2}\right)\left\{a^{2}+a(b+\beta)+b \beta\right\}-(n+1) a\left(a^{2}-a^{2}\right)(b-\beta)=0$.

Adding (5) and (6) we get

$$
\left(\alpha^{2}+a^{2}\right)\left(a^{2}+b \beta\right)=0
$$

the conjugate of which is $\left(a^{2}+a^{2}\right)\left(a^{2}+b \beta\right)=0$.

By subtraction we get

$$
\left(a^{2}+a^{2}\right)\left(a^{2}-a^{2}\right)=0 .
$$

In order that this equation be fulfilled we must have either

$$
a^{2}+a^{2}=0 \text { or } a^{2}-a^{2}-0 .
$$

We shall examine each of those conditions for the curve being algebraic.

First case, $a^{2}+a^{2}=0$.

(5) then gives

$$
\begin{gathered}
\left(a^{2}-a^{2}\right)(b-\beta)=0 \\
a^{2}-a^{2}=0 \text { or } b-\beta=0 .
\end{gathered}
$$

hence either

The first of these is impossible for taken along with $a^{2}+a^{2}=0$, it would give zero for $a$ and $a$.

We therefore must take $b-\beta=0$, or $b=\beta$, so that the curve (4) is

$$
x+i y=\int \frac{(z-a)(z+a)}{(z-a)^{2}(z+a)^{2}} d z
$$

which, see the foot-note on page 19 , is the lemniscate. 
Second case, $a^{2}-a^{2}=0$ or $a^{2}=a^{2}$.

But the arc $\quad s=\int \frac{d z}{\sqrt{\left(z^{2}-a^{2}\right)\left(z^{2}-a^{2}\right)}}=\int \frac{d z}{z^{2}-a^{2}}$

which is no longer an elliptic integral of the first kind, and therefore we need not further discuss the case.

\section{VII.}

The most general solutions, however, of the problem we are investigating have been obtained by employing the Weierstrassian notation for elliptic functions.

The transition from the older notation to that now almost universally employed can be very briefly stated for our present purpose.

Oall the arc of a curve $u$, then up to the present we have used the relation

$$
\sqrt{d x^{2}+d y^{2}}=d u=\frac{d z}{\sqrt{a_{0} z^{4}+4 a_{1} z^{3}+6 a_{2} z^{2}+4 a_{3} z+a_{4}}}
$$

But the elliptic function $z$ of the argument $u$ defined as above by the equation

$$
\left(\frac{d z}{d u}\right)^{2}=a_{0} z^{4}+4 a_{2} z^{3}+6 a_{2} z^{2}+4 a_{5} z+a_{4}
$$

can be transformed into Weierstrass's elliptic function $\mathrm{p} u$ of the argument $u$ defined by the equation

$$
\left(\mathrm{p}^{\prime} u\right)^{2} \text { or }\left(\frac{d \cdot \mathrm{p} u}{d u}\right)^{2}=4 \mathrm{p}^{3} u-g_{2} \mathrm{p} u-g_{3}
$$

( $\mathrm{p}^{3} u$ denotes the cube of $\mathrm{p} u$ ) where $g_{2}$ and $g_{8}$ are the invariants of the 2nd and 3rd degrees of the quadric function

$$
\begin{aligned}
& a_{0} z^{4}+4 a_{2} z^{3}+6 a_{2} z^{2}+4 a_{9} z+a_{4} . \\
& \int \sqrt{d x^{2}+d y^{3}}=u=\int \frac{d(\mathrm{p} u)}{\sqrt{4 \mathrm{p}^{3} u-g_{2} \mathrm{p}^{2} u-g_{3}}}
\end{aligned}
$$

then the arc $u$ of the curve is, in the new notation, an elliptic integral of the first kind. 
Furthermore, if $2 \omega$ and $2 \omega^{\prime}$ be the two fundamental real and imaginary periods respectively of this doubly periodic function $\mathrm{pu}$, and $2 \omega^{\prime \prime}$ their sum, and if we put $e_{1}$ for p $\omega, e_{2}$ for $p \omega^{\prime \prime}$, and $e_{3}$ for p $\omega^{\prime}$, then

$$
\left(\mathrm{p}^{\prime} u\right)^{2}=4\left(\mathrm{p} u-e_{1}\right)\left(\mathrm{p} u-e_{2}\right)\left(\mathrm{p} u-e_{3}\right)
$$

Comparing this with (1) we see that

$$
\begin{aligned}
& 0=e_{1}+e_{2}+e_{3} \\
& g_{2}=-4\left(e_{2} e_{3}+e_{3} e_{1}+e_{1} e_{2}\right) \\
& g_{3}=4 e_{1} e_{2} e_{3} .
\end{aligned}
$$

From $p u$ we define the $\sigma u$ function of Weierstrass by the equation

$$
\frac{d^{2} \log \sigma u}{d u^{2}}=-\mathrm{p} u
$$

with the additional equations

$$
\sigma(0)=0, \sigma^{\prime}(0)=1, \sigma^{\prime \prime}(0)=0
$$

for the determination of the constants of integration. *

* The best summary of Weierstrass's functions is contained in H. A. Schwarz's Formeln und Lehrsätze zum Gebrauche der elliptischen Functionen, nach Vorlesungen und Aufzeichnungen des Herrn $K$. Weierstrass (2nd edition, 1893). The theory of these functions is developed in Halphen's Traité des Fonctions Elliptiques. Weierstrass's Memoirs have been collected and published in Mathematische Werke von Karl Weierstrass (Berlin, Bd. I. 1894; Bd. II. 1895). In Bd. II., pp. 245.309, are two articles $Z$ ur Theorie der elliptischen Functionen. No better account of the methods of Legendre, Jacobi and Weierstrass can be found than that in Müller's edition of Enneper's Elliptische Functionen, Theorie und Geschichte (Halle, 1890). It contains also for the student of the subject a great mass of bibliographical details. The best Memoirs in English on Weierstrass's methods are three by A. L. Daniels in vols. vi. and vii. (1884 and 1885) of The American Joumal of Mathematicy. Dr A. R. Forayth has a Memoir on the same subject in vol. xxii. (1887) of The Quarterly Journal of Mathematics. In Greenhill's Elliptic Functions some parts of the modern notation are developed alongside of the old. In chap. vii. of A. C. Dixon's excellent little book on Elliptic Functions, there is a very brief sketch of the $p$ - and $\zeta$ - functions (Halphen uses the gymbol $s u$ for $\frac{d}{d u} \log \sigma u$ or $\frac{\sigma^{\prime}}{\sigma} u$ ). Harkness and Morley in chap. vii. of their Treatise on the Theory of Functions devote about 60 pages to the $\mathrm{p}-, \sigma-$, and $\zeta$ - functions. 


\section{VIII.}

KIRPERT* by using the Weierstrassian notation was able to obtain a more general solution than Serret's. Indeed, we shall show that Kiepert discovered a class of curves, whose arcs are elliptic integrals of the first kind, which includes Serret's infinity of curves as a particular case.

If $\phi(u)$ be a doubly periodic function of the argument $u$ of the $\gamma^{2 h}$ degree having $2 \omega$ and $2 \omega^{\prime}$ as its primary periods then we shall in what follows use two methods of expressing this function by means of the $\sigma$ - function.

First, $\uparrow$ it is always possible to find $2 r+1$ quantities $\mathrm{C}, a_{1}, a_{2}, \ldots . . \quad a_{r}, b_{1}, b_{2}, \ldots . . \quad b_{r}$, so that

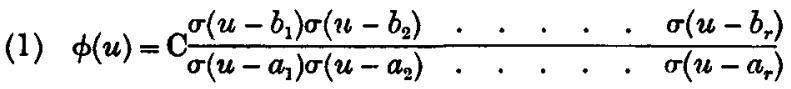

where $\mathrm{C}$ is a constant, and $b_{1}, b_{2}$, . . . . . . $b_{r}$ are the values of $u$ for which $\phi(u)$ vanishes, and $a_{1}, a_{2}$, . . . . . $a_{r}$ are the values of $u$ for which $\phi(u)$ is infinitely great.

It can be proved that as a consequence of (1)

(2) $a_{1}+a_{2}+\ldots . . .++a_{r}=b_{1}+b_{2}+\ldots . . .+b_{r}$

Conversely, if $a_{1}$, . . . . . $a_{r}, b_{1}$. . . . . $b_{r}$ satisfy equation (2), then every doubly periodic function of the $r^{\text {th }}$ degree can be expressed as in (1).

Secondly, Kiepert makes very frequent use of another mode of expressing any doubly periodic function $\phi(u)$ of the $i^{\text {th }}$ degree, viz., a modified form of the expression given at p. 20 of the Formeln und Lehrsätze

Among the values $a_{1}, a_{2}, \ldots \ldots a_{\text {r. of }} u$ for which $\phi(u)$ is infinite there may be only $m$ different from each other, viz.,

$$
a_{1}, a_{2} \ldots \ldots a_{m},
$$

and these may occur respectively $r_{1}, r_{2} \ldots \ldots r_{m}$ times

* Inaugural Dissertation De curvis quarum arcus integralibus ellipticis primi generis exprimuntur (Berlin 1870); Ueber Curvent deren Bogen ein elliptisches Integral erster Gattung ist (Crelle's Journal, vol. lxxix., 1875 ; and Berichte der naturforschenden Gesellschaft zu Freiburg, 1876).

† Of. Formeln, p. 15, or Halphen's F. E., rol. I, p. 213. 
i.e., the infinities may respectively be of the orders

so that

$$
r_{1}, r_{2} \ldots r_{m} \text {, }
$$

Now, in the development of $\phi(u)$ according to powers of $u-a$, the sum of the terms with negative exponents may be, say,

(3) $c_{1},{ }_{1}\left(u-a_{1}\right)^{-1}+c_{1},{ }_{2}\left(u-a_{1}\right)^{-2}+\ldots$

$$
\cdots+c_{1, r_{1}-1}\left(u-a_{1}\right)^{-r_{1}+1}+c_{1, r_{1}}\left(u-a_{1}\right)^{-r_{1}}
$$

with similar series for $u-a_{2}$, etc., and lastly

$$
\begin{gathered}
c_{m},{ }_{1}\left(u-a_{m}\right)^{-1}+c_{m},{ }_{2}\left(u-a_{m}\right)^{-2}+\ldots \\
\ldots+c_{m, r_{m-1}}\left(u-a_{m}\right)^{-r_{m}+1}+c_{m, r_{m}}\left(u-a_{m}\right)^{-r_{m}} .
\end{gathered}
$$

From these expressions it is evident that if we put

$$
\phi\left(u, a_{1}\right) \text { for } \sum_{\nu=1}^{r_{1}} \frac{(-1)^{\nu-1}}{(\nu-1) !} c_{1}, \nu \frac{d^{\nu} \log \sigma\left(u-a_{1}\right)}{d u^{\nu}}
$$

$\phi\left(u, a_{2}\right), \quad \phi\left(u, a_{3}\right) \ldots \ldots \phi\left(u, a_{m}\right) \quad$ having similar meanings, then

(4) $\phi(u)-\phi\left(u, a_{1}\right)-\phi\left(u, a_{2}\right) \ldots \ldots-\phi\left(u, a_{m}\right)=\chi(u)$, say, where $\chi(u)$ must by hypothesis be a function which cannot become infinite for any finite value of $u$.

If we write down the expanded form of (4) we see that

$$
c_{1}{ }_{1}+c_{2},{ }_{1}+\ldots .+c_{m},{ }_{1}=0 .
$$

Also, on differentiating each side of (4) we obtain that the derivative of $\chi(u)$ is doubly periodic since the derivatives $\phi^{\prime}$ are so. But every doubly periodic function must have infinite values, therefore $\chi^{\prime}(u)$ cannot be a function of $u$, it can at most be a constant. If we put in turn $u+2 \omega$ and $u+2 \omega^{\prime}$ for $u$ in the differential of each side of (4), we shall find that this constant is zero. Hence, since $\chi^{\prime}(u)$ is zero, $\chi(u)$ is constant, say, $c_{0}$.

Wherefore we may write (4) as follows:

$$
\begin{aligned}
& \phi(u)=c_{0}+\phi\left(u, a_{1}\right)+\phi\left(u, a_{2}\right)+\ldots \ldots+\phi\left(u, a_{m}\right), \text { or } \\
&(5) \phi(u)=c_{0}+\sum_{\nu=1}^{r_{1}} c_{1, \nu} \frac{d^{\nu} \log \sigma\left(u-a_{1}\right)}{d u^{\nu}}+\sum_{\nu=1}^{r_{2}} c_{2, \nu} \frac{d^{\nu} \log \sigma\left(u-a_{2}\right)}{d u^{\nu}}+\ldots \\
& \ldots+\sum_{\nu=1}^{r_{m}} c_{m, \nu} \frac{d^{\nu} \log \sigma\left(u-a_{m}\right)}{d u^{\nu}}
\end{aligned}
$$


where, as we have seen,

$$
c_{1},_{1}+c_{2},{ }_{1}+\ldots+c_{m}, 1=0 .
$$

Comparing the right-hand side of (5) with the expression given above for $\phi\left(u, a_{1}\right)$, it will be seen that we have omitted the part $\frac{(-1)^{\nu-1}}{(\nu-1) !}$ in the 2nd, 3rd, 4th, etc., terms. Of course this is permissible since the factor omitted only affects the coefficients.

Now, using (5), let the equation of the curves we are going to investigate be

(7) $x+i y=c_{0}+\sum_{\nu=1}^{r_{1}} c_{1, \nu} \frac{d^{\nu} \log \sigma\left(u-a_{1}\right)}{d u u^{\nu}}+\ldots$

$$
\ldots+\sum_{\nu=1}^{r_{m}} c_{m, \nu} \frac{d^{\nu} \log \sigma\left(u-a_{m}\right)}{d u^{\nu}}
$$

with the condition (6) amongst the coefficients.

We have to find the condition that those curves be algebraic, and to find a method of obtaining the modulus of the elliptic integral of the first kind which the arcs of the curves represent.

Differentiating (7) we get

(8) $\frac{d x+i d y}{d u}$

$=f(u)=\sum_{\nu=1}^{r_{1}} c_{1, \nu} \frac{d^{\nu+1} \log \sigma\left(u-a_{1}\right)}{d u^{\nu+1}}+\ldots+\sum_{\nu=1}^{r_{m}} c_{m, \nu} \frac{d^{\nu+1} \log \sigma\left(u-a_{m}\right)}{d u^{\nu+1}}$

From (1) we get similarly

(9) $\frac{d x+i d y}{d u}$

$=f(u)=\mathrm{C} e^{c u t} \frac{\Pi \sigma(u-b)}{\sigma\left(u-a_{1}\right)^{r_{1}+1} \sigma\left(u-a_{2}\right)^{r_{2}+1} \ldots \sigma\left(u-a_{m}\right)^{r_{m}+1}}$

$\therefore \quad(10) \frac{d x-i d y}{d u}$

$=\Gamma e^{\gamma u} \frac{\Pi \sigma(u-\beta)}{\sigma\left(u-a_{1}\right)^{r_{1}+1} \sigma\left(u-a_{2}\right)^{r_{2}+1} \ldots: \sigma\left(u-a_{n n}^{\prime} r_{m}+1\right.}$

3 Vol. 15 
where $\quad \Gamma, \gamma, \beta, a_{1}, a_{2}, \ldots \alpha_{m}$ are the conjugate complex quantities of $\mathrm{C}, c, b, a_{1}, a_{2}, \ldots a_{m}$ respectively.

If the argument $u$ (which is an elliptic integral of the first kind, vide p. 25) represent an arc of the curves, then

$$
d x^{2}+d y^{2}=d u^{2} \text { or } \frac{d x+i d y}{d u} \quad \frac{d x-i d y}{d u}=1 .
$$

Therefore from (9) and (10) it follows that

(11) $\left\{\begin{array}{l}\mathrm{C} \Gamma=1, c+\gamma=0 \\ \Pi \sigma(u-b)=\sigma\left(u-a_{1}\right)^{r_{1}+1} \sigma\left(u-a_{2}\right)^{r_{2}+1} \ldots \sigma\left(u-a_{m}\right)^{r_{m}+1}\end{array}\right.$

are the sufficient and necessary conditions that the curves (7) expressed by the integral $\int f(u) d u$ be algebraic.

These conditions are satisfied if (since $b_{1}, b_{2} \ldots$ are the zeros of $f(u)$ )

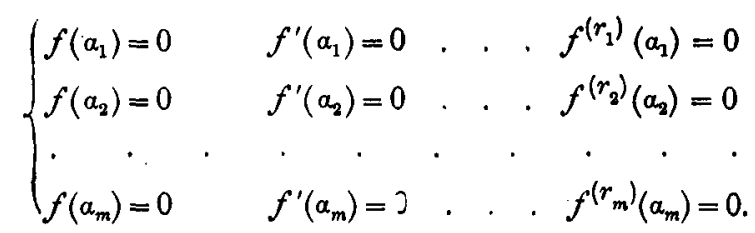

We have here $m+r_{1}+r_{2}+\ldots+r_{m}$ or $m+r$ equations of condition, which can be satisfied since we have the $m$ quantities $a_{1}, a_{2} \ldots a_{m}$, and the $r$ coefficients $c$ at our disposal. From the former we get as in Serret's case the modulus of the elliptic function. In trying to find the modulus there are in practice very great, if not sometimes insuperable, slgebraic difficulties; but we can generally simplify the process, for $x+i y=\int f(u) d u$ will frequently lead to an expression containing various $\sigma$ - functions and their logarithmic derivatives, and from the properties of such functions we can get many of the conditions to disappear, making it easier to find quantities to satisfy the remaining conditions. We shall illustrate this by three examples. 


\section{First Example.}

(13) Let $\frac{d x+i d y}{d u}=f(u)$

$$
=\sum_{\nu=1}^{m} c_{1, \nu} \frac{d^{2 \nu+1} \log \sigma\left(u-a_{1}\right)}{d u^{2 \nu+1}}+\sum_{\nu=1}^{n} c_{2, \nu} \frac{d^{2 \nu+1} \log \sigma\left(u-a_{2}\right)}{d u^{2 \nu+1}}
$$

with the further condition that $a_{1}, a_{2}$ and their conjugates $a_{1}, a_{2}$ are such that their differences $a_{1}-a_{1}, a_{1}-a_{2}, a_{2}-a_{1}, a_{2}-a_{2}$ are semiperiods.

Comparing (13) with (8) we see that the conditions corresponding to (12) for the curves being algebraic are

$$
\left\{\begin{array}{l}
f\left(a_{1}\right)=0, \quad f^{\prime}\left(a_{1}\right)=0, \quad f^{\prime \prime}\left(a_{1}\right)=0 \ldots f^{(2 m)}\left(a_{1}\right)=0 \\
f\left(a_{2}\right)=0, \quad f^{\prime}\left(a_{2}\right)=0, \quad f^{\prime \prime}\left(a_{2}\right)=0 \ldots f^{(2 n)}\left(a_{2}\right)=0 .
\end{array}\right.
$$

But, by the properties of the $\sigma$ functions, if $\lambda$ be a positive integer, then * $\frac{d^{2 \lambda+1}}{d u^{2 \lambda+1}} \log \sigma u=0$, if $u=\omega, \omega^{\prime}$, or $\omega^{\prime \prime}\left(\omega^{\prime \prime}=\omega+\omega^{\prime}\right)$.

Hence it is evident from (13) that $f\left(a_{1}\right), f\left(a_{2}\right)$ and all their even derivatives vanish, so that in (14) only the following $m+n$ conditions remain to be satisfied :-

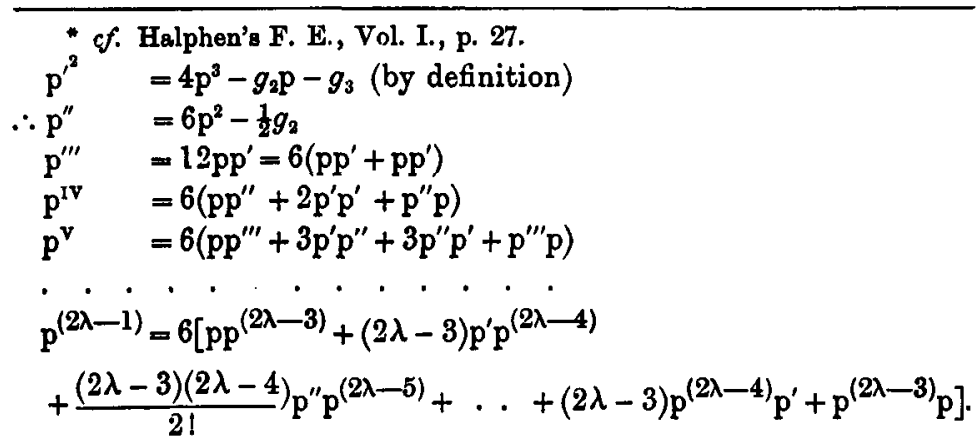

From this it can be seen that since $\mathrm{p}^{\prime} \omega=0, \mathrm{p}^{\prime} \omega^{\prime}=0, \mathrm{p}^{\prime} \omega^{\prime \prime}=0^{\prime}$ (see Formeln, p. 11) $\therefore \mathrm{p}^{\prime \prime \prime} u, \mathrm{p}^{\vee} u$, and all the other odd derivatives of pu vanish for $u=\omega, \omega^{\prime}$, or $\omega^{\prime \prime}$. But

$$
\frac{d^{2} \log \sigma u}{d u^{2}}=-\mathrm{p} u \quad \therefore \frac{d^{2 \lambda+1} \log \sigma u}{d u^{2 \lambda+1}}=-\mathrm{p}^{(2 \lambda-1)} u=0
$$

when $u=\omega, \omega^{\prime}$, or $\omega^{\prime \prime}$. 


$$
\left\{\begin{array}{l}
f^{\prime}\left(a_{1}\right)=0, f^{\prime \prime \prime}\left(a_{1}\right)=0 \ldots f^{(2 n-1)}\left(a_{1}\right)=0 \\
f^{\prime}\left(a_{2}\right)=0, \quad f^{\prime \prime \prime}\left(a_{2}\right)=0 \ldots f^{(2 n-1)}\left(a_{2}\right)=0
\end{array}\right.
$$

which are linear and homogeneous equations among the $m+n$ coefficients $c$, whence the conditions that the curves be algebraic can be satisfied.

We may satisfy the given condition that $a_{1}-a_{1}$, etc., be semiperiods in two ways, viz.,

$$
\left.\begin{array}{l}
a_{1}=\frac{\omega}{2}-\frac{\omega^{\prime}}{2}, a_{2}=-\frac{\omega}{2}+\frac{\omega^{\prime \prime}}{2} \\
a_{1}=\frac{\omega}{2}+\frac{\omega^{\prime}}{2}, a_{2}=-\frac{\omega}{2}-\frac{\omega^{\prime}}{2}
\end{array}\right\} \text { or }\left\{\begin{array}{l}
a_{1}=-\frac{\omega^{\prime}}{2}, a_{2}=-\omega+\frac{\omega^{\prime}}{2} \\
a_{1}=\frac{\omega^{\prime}}{2}, a_{2}=-\omega-\frac{\omega^{\prime *}}{2}
\end{array}\right.
$$

so that from (13) we get two functions $f(u)$ to satisfy the case we are discussing, viz.,

$$
\text { (16) } \begin{aligned}
f(u)= & \sum_{\nu=1}^{m} c_{1}, \frac{d^{2 \nu+1}}{d u^{2 \nu+1}} \log \sigma\left(u-\frac{\omega}{2}+\frac{\omega^{\prime}}{2}\right) \\
& +\sum_{\nu=1}^{n} c_{2}, \frac{d^{2 \nu+1}}{d u^{2 \nu+1}} \log \sigma\left(u+\frac{\omega}{2}-\frac{\omega^{\prime}}{2}\right)^{\dagger}
\end{aligned}
$$

and $\quad f(u)=\sum_{\nu=1}^{m} c_{1}, \frac{d^{2 \nu+1}}{d u^{2 \nu+1}} \log \sigma\left(u+\frac{\omega^{\prime}}{2}\right)$

$$
+\sum_{\nu=1}^{n} c_{2}, \nu \frac{d^{2 \nu+1}}{d u^{2 \nu+1}} \log \sigma\left(u+\omega-\frac{\omega^{\prime}}{2}\right)
$$

* We get other two sets of values that satisfy the given conditions by changing the sign of $\frac{\omega^{\prime}}{2}$ in each of the above, but of course these values would just be the conjugates of the foregoing.

+ A little consideration will show that the curves represented by this equation (which is erroneously stated by Kiepert on page 11 of his disserta. tion) embrace all Serret' $\times$ curves $\frac{d x+i d y}{d z}=\frac{(z-a)^{m}(z+a)^{n}}{(z-a)^{m+1}(z+a)^{n+1}}$. For in the latter we see that there are two infinities which are the same but of opposite signs, viz., $z=+a$, and $z--a$. So also in the curves represented by (16), remembering that $\frac{d^{2} \log \sigma u}{d u^{2}}=-p u$, and that $\mathrm{p}(0)=\infty$, and $\mathrm{p}^{\prime}(0)=\infty$, we see that the infinities are $u=\frac{\omega}{2}-\frac{\omega^{\prime}}{2}$ and $\imath=-\frac{\omega}{2}+\frac{\omega^{\prime}}{2}$, i.e., they differ only in sign. 


\section{Stcond Example.}

Next take an example suggested by the two equations just given.

(17) Let $\frac{d x+i d y}{d u}=f(u)$

$$
\begin{aligned}
& =\sum_{\nu=1}^{m} c_{1, \nu} \frac{d^{2 \nu}}{d u^{2 \nu}}\left\{\log \sigma\left(u+\frac{\omega^{\prime}}{2}\right)-\log \sigma\left(u-\omega+\frac{\omega^{\prime}}{2}\right)\right\} \\
& +\sum_{\nu=1}^{n} c_{2}, \nu \frac{d^{2 \nu+1}}{d u^{2 \nu+1}}\left\{\log \sigma\left(u+\frac{\omega}{2}-\frac{\omega^{\prime}}{2}\right)-\log \sigma\left(u-\frac{\omega}{2}-\frac{\omega^{\prime}}{2}\right)\right\} .
\end{aligned}
$$

Comparing this with the general equation (8) we see that

$$
\begin{cases}r_{1}=r_{2}=2 m-1, & r_{3}=r_{4}=2 n \\ a_{1}=-\frac{\omega^{\prime}}{2}, a_{2}=\omega-\frac{\omega^{\prime}}{2} & a_{3}=-\frac{\omega}{2}+\frac{\omega^{\prime}}{2}, a_{4}=\frac{\omega}{2}+\frac{\omega^{\prime}}{2} \\ a_{1}=+\frac{\omega^{\prime}}{2}, a_{2}=\omega+\frac{\omega^{\prime}}{2} & a_{3}=-\frac{\omega}{2}-\frac{\omega^{\prime}}{2}, a_{4}=\frac{\omega}{2}-\frac{\omega^{\prime}}{2} .\end{cases}
$$

For the present case the equations of condition corresponding to (12) are

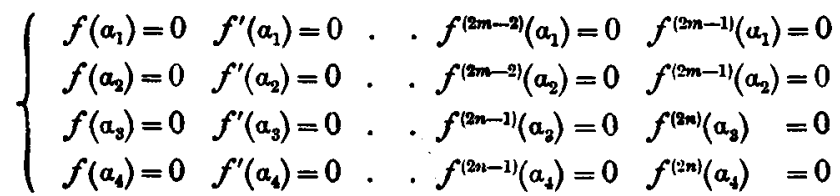

If the argument $u$ is increased by $\omega$, then

$$
\log \sigma\left(u+\frac{\omega^{\prime}}{2}\right)-\log \sigma\left(u-\omega+\frac{\omega^{\prime}}{2}\right)+\log \sigma\left(u+\frac{\omega}{2}-\frac{\omega^{\prime}}{2}\right)-\log \sigma\left(u-\frac{\omega}{2}-\frac{\omega^{\prime}}{2}\right)
$$

becomes

$$
\log \sigma\left(u+\omega+\frac{\omega^{\prime}}{2}\right)-\log \sigma\left(u+\frac{\omega^{\prime}}{2}\right)+\log \sigma\left(u+\frac{3 \omega}{2}-\frac{\omega^{\prime}}{2}\right)-\log \sigma\left(u+\frac{\omega}{2}-\frac{\omega^{\prime}}{2}\right) .
$$

But, since $\pm 2 \omega$ is one of the periods of the function, this is equal to

$$
\log \sigma\left(u-\omega+\frac{\omega^{\prime}}{2}\right)-\log \sigma\left(u+\frac{\omega^{\prime}}{2}\right)+\log \sigma\left(u-\frac{\omega}{2}-\frac{\omega^{\prime}}{2}\right)-\log \sigma\left(u+\frac{\omega}{2}-\frac{\omega^{\prime}}{2}\right)
$$


which is just (19) with a negative sign.

But

$$
\begin{aligned}
& a_{2}=a_{1}+\omega, \quad \text { and } a_{4}=a_{3}+\omega \\
& \therefore \quad f\left(a_{2}\right)=-f\left(a_{1}\right) \text { and } f\left(a_{4}\right)=-f\left(a_{3}\right)
\end{aligned}
$$

so that if $f\left(a_{1}\right)$ vanishes so must $f\left(a_{2}\right)$, and if $f\left(a_{3}\right)$ vanishes so must $f\left(a_{4}\right)$, and the same is true of their derivatives, so that in (18) we may omit the 2 nd and 4 th rows, and thus the number of necessary conditions is reduced by one-half.

Again, both parts of the right-hand side of (17) belong to the type

$$
\log \sigma u-\log \sigma(u-\omega) \text {. }
$$

But by the properties of the $\sigma$ - functions

(20) $\frac{d^{2 \lambda+1}}{d u^{2 \lambda+1}}\{\log \sigma u-\log \sigma(u-\omega)\}$ vanishes if $u= \pm \omega^{\prime}, \pm \omega \pm \omega^{\prime}$ and

(21) $\frac{d^{2 \lambda}}{d u^{2 \lambda}}\{\log \sigma u-\log \sigma(u-\omega)\}$ vanishes if $u= \pm \frac{\omega}{2}, \pm \frac{\omega}{2} \pm \omega^{\prime}$.

$$
\begin{aligned}
& \text { But } a_{1}-a_{1}=\omega \\
& a_{1}-a_{2}=-\omega+\omega^{\prime} \quad \therefore \text { all odd derivatives of } f\left(a_{1}\right) \text { vanish } \\
& a_{1}-a_{3}=\frac{\omega}{2}\left\{\begin{array}{l}
\text { [the first half by condition (20), } \\
\text { and the second half by (21)]. }
\end{array}\right. \\
& a_{1}-a_{4}=-\frac{\omega}{2} \\
& \text { Also } \left.\begin{array}{rl}
a_{3}-a_{1}=-\frac{\omega}{2} \\
a_{3}-a_{2}=-\frac{3 \omega}{2} \\
a_{3}-a_{3}=-\omega^{\prime} \\
a_{3}-a_{4}=-\omega-\omega^{\prime}
\end{array}\right\} \begin{array}{r} 
\\
\text { vanish [the first half by }(21) \text {, } \\
\text { and the second half by }(20)] .
\end{array}
\end{aligned}
$$

Hence, finally, taking all these into account, (18) reduces to only $m+n$ conditions, viz.,

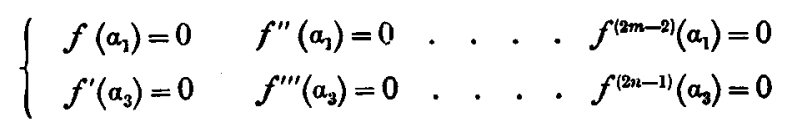

which can be satisfied since we have the $m+n$ coefficients $c$ at our disposal. 
Kiepert works out at great length the particular case of the group of curves (17) when $m=n=1$.

The equation he finally gets is

$$
54\left(x^{4}-y^{4}\right)=36\left(x^{2}+y^{2}\right)^{3}+18\left(x^{2}+y^{2}\right)-\sqrt{ } 3
$$

whose shape is given in Figure 14.

\section{Third Example.}

As another group of the infinity of curves discovered by himself Kiepert takes

(22) $\frac{d x+i d y}{d u}=\sum_{\lambda=0}^{n_{1}} c_{1, \lambda} \psi^{(\lambda)}\left(u+\beta_{1} i\right)+\sum_{\lambda=0}^{n_{2}} c_{1, \lambda} \psi^{(\lambda)}\left(u+\beta_{2} i\right)+\ldots$

$$
\text { . . }+\sum_{\lambda=0}^{n_{k}} c_{1, \lambda} \psi^{(\lambda)}\left(u+\beta_{k} i\right)
$$

where $\beta_{1}, \beta_{2}$. . . . $\beta_{k}$ are real quantities, and for brevity $\psi^{(\lambda)}(u)$ represents

$$
\begin{gathered}
\frac{d^{(\lambda+2)}}{d u^{(\lambda+2)}}\left\{\log \sigma u+\epsilon \log \sigma\left(u+\frac{1}{r} 2 \hat{\omega}\right)+\epsilon^{2} \log \sigma\left(u+\frac{2}{r} 2 \hat{\omega}\right)+\ldots\right. \\
\left.\ldots+\epsilon^{r-1} \log \sigma\left(u+\frac{r-1}{r} 2 \hat{\omega}\right)\right\} \\
(\lambda=0,1,2, \ldots . .)
\end{gathered}
$$

where $2 \hat{\omega}$ is any period of the elliptic function, and $\epsilon$ is the $r^{\text {th }}$ root of unity.

He shows that the necessary conditions can be satisfied that (22) may be algebraic.

He examines in detail the case where $r=3 ; n_{1}=0, k=1$, and $2 \hat{\omega}=-4 \omega+2 \omega^{\prime}$.

$$
\begin{aligned}
& \therefore \frac{d x+i d y}{d u}=\psi\left(u+\frac{2 \omega}{3}-\frac{2 \omega^{\prime}}{3}\right) \\
& =-\frac{d^{2}}{d u^{2}}\left[\log \sigma\left(u+\frac{2 \omega}{3}-\frac{2 \omega^{\prime}}{3}\right)+\epsilon \log \sigma\left(u+\frac{2 \omega}{3}-\frac{2 \omega^{\prime}}{3}+\frac{1}{3}\left(-4 \omega+2 \omega^{\prime}\right)\right)\right. \\
& \left.+\epsilon^{2} \log \sigma\left(u+\frac{2 \omega}{3}-\frac{2 \omega^{\prime}}{3}+\frac{2}{3}\left(-4 \omega+2 \omega^{\prime}\right)\right)\right] \\
& =\mathrm{p}\left(u+\frac{2 \omega}{3}-\frac{2 \omega^{\prime}}{3}\right)+\epsilon \mathrm{p}\left(u-\frac{2 \omega}{3}\right)+\epsilon^{2} \mathrm{p}\left(u+\frac{2 \omega^{\prime}}{3}\right) \text {. }
\end{aligned}
$$


He shows that the cartesian equation of the curre is $\left(x^{2}+y^{2}\right)^{3}-y\left(y^{2}-3 x^{2}\right)=0$ and its polar equation $r^{3}=\cos 3 \theta^{*}$

The curve consists of 3 equal parts as in Figure 15. Similarly all the algebraic curves (22) are composed of $r$ equal parts.

IX.

The weakness of both Serret's and Kiepert's methods for plane curves is that they cannot be extended to space curves. As we have seen, they both start from the expression $x+i y$; they form an elliptic function for this, and find the conditions that the curve be algebraic, i.e. that $\left(\frac{d x}{d u}\right)^{2}+\left(\frac{d y}{d u}\right)^{2}=$ constant. Of course such a method is not applicable to curves of double curvature. To get a method applicable at once to curves of single and double curvature Kiepert's method has been somewhat modified by $\mathbf{R}$. von LiLignthaL in a dissertation entitled Zur Theorie der Curven deren Bogenlänge ein elliptisches Integral erster Art ist (Berlin, 1882). He proceeds thus: he puts the co-ordinates $x$ and $y$ (and $z$ also for a space curve) equal to certain elliptic functions. Then he forms the expression for $\left(\frac{d x}{d u}\right)^{2}+\left(\frac{d y}{d u}\right)^{2}$ or $\left(\frac{d x}{d u}\right)^{2}+\left(\frac{d y}{d u}\right)^{2}+\left(\frac{d z}{d u}\right)^{2}$ and finds the condition that these sums are constant, i.e. that the co-efficients of the infinite terms vanish.

* Observe the analogy of this curve to the lemniscate $r^{2}=\cos 2 \theta$, which of course consists of two equal loops.

We can very easily reduce the expression for an arc $u$ of $r^{3}=\cos 3 \theta$ to an elliptic integral of the first kind; for

$$
d u=\sqrt{d r^{2}+r^{2} d \theta^{2}}=\frac{d r^{2}}{\sqrt{1-r^{6}}}
$$

Take $r^{2}=\frac{1}{\rho}, \therefore$ the above becomes

$$
d u=-\frac{d \rho}{\sqrt{4 \rho^{3}-4}} \text { or } u=-\int \frac{d \rho}{\sqrt{4 \rho^{3}-4}}
$$

Kiepert shows in Crelle's Joumal, Vol. LXXIV. (1872), how to divide an arc of the curve $r^{3}=\cos 3 \theta$ into $7,13,19,31$ or, generally, $6 q+1$ equal parts. 
Lilienthal illustrates his method by applying it to the case of the plane lemniscate and the "spherical" lemniscate, and in the latter part of his paper he discusses the connection between spherical curves whose arcs are elliptic integrals of the first kind and surfaces of minimum area for given perimeter (Minimalf(üchen).*

Starting with the lemniscate, he puts

$-x=$

$\frac{\mathbf{A} d^{2} \log \sigma(u-a)}{d u^{2}}+\frac{\mathbf{A}^{\prime} d^{2} \log \sigma\left(u-a^{\prime}\right)}{d u^{2}}+\frac{\mathbf{A}_{2} d^{2} \log \sigma\left(u-a_{1}\right)}{d u^{2}}+\frac{\mathbf{A}_{1}^{\prime} d^{2} \log \sigma\left(u-a_{1}^{\prime}\right)}{d u^{2}}$

or

(1) $\left\{\begin{array}{l}x=\mathrm{Ap}(u-a)+\mathrm{A}^{\prime} \mathrm{p}\left(u-a^{\prime}\right)+\mathrm{A}_{1} \mathrm{p}\left(u-a_{1}\right)+\mathrm{A}_{1}^{\prime} \mathrm{p}\left(u-a_{1}^{\prime}\right) \\ y=\mathrm{B} \mathrm{p}(u-a)+\mathrm{B}^{\prime} \mathrm{p}\left(u-a^{\prime}\right)+\mathrm{B}_{1} \mathrm{p}\left(u-a_{1}\right)+\mathrm{A}_{1}^{\prime} \mathrm{p}\left(u-a_{1}^{\prime}\right)\end{array}\right.$

where $a^{\prime}, a_{1}^{\prime}, A^{\prime}, A_{1}^{\prime}, B^{\prime}, B_{1}^{\prime}$ are conjugate respectively to

$a, a_{1}, \mathrm{~A}, \mathrm{~A}_{1}, \mathrm{~B}, \mathrm{~B}_{1}$.

Our problem is to determine those quantities so that $\left(\frac{d x}{d u}\right)^{2}+\left(\frac{d y}{d u}\right)^{2}=$ constant

We have

(2) $\frac{d x}{d u}=\mathrm{Ap}^{\prime}(u-a)+\mathrm{A}^{\prime} \mathrm{p}^{\prime}\left(u-a^{\prime}\right)+\mathrm{A}_{1} \mathrm{p}^{\prime}\left(u-a_{1}\right)+\mathrm{A}_{1}^{\prime} \mathrm{p}^{\prime}\left(u-a_{1}^{\prime}\right)$.

If we develop this in the neighbourhood of $u=a$ we get $\dagger$

* For the properties of minimalfächen see Todhunter's History of the Progress of the Calculus of Variations (1861); Riemann's Memoir Ueber die Fläche vom kleinsten Inhalt bie gegebener Begrenzung, revised by $\mathbf{K}$. Hattendorff, Bd. 13 der Abhandlungen der Königlichen Gesellschaft der Wissenschaften zu Gottingen (1867); and particularly H. A. Schwarz's article Miscellen aus dem Gebiete der Minimalfä̈chen, at pp. 168-189, vol. i., of his Mathematische Abhandlungen (Berlin, 1890).

† For the development of $\mathrm{p}^{\prime}(u-a)$ see Formeln, p. 11, and for the other three terms we use the theorem that if $f(u)$ be an elliptic function of $u$, then its development in the neighbourhood of $u=a$ is

$$
\begin{aligned}
& f(u)=f(a)+(u-a) f^{\prime}(a)+\frac{(u-a)^{2}}{2 !} f^{\prime \prime}(a)+\frac{(u-a)^{3}}{3 !} f^{\prime \prime \prime}(a)+\ldots \\
& \ldots+\frac{(u-a)^{n}}{n !} f^{(n)}(a) . \quad(C f . \text { Forsyth's Theory of Functions of a Complex }
\end{aligned}
$$

Variable, p. 50). 


$$
\begin{aligned}
& \frac{d x}{d u}=-\frac{2 \mathrm{~A}}{(u-a)^{3}}+\frac{\mathbf{A} g_{2}}{10}(u-a)+\ldots \\
& +\mathrm{A}^{\prime} \mathrm{p}^{\prime}\left(a-a^{\prime}\right)+(u-a) \mathrm{A}^{\prime} \mathrm{p}^{\prime \prime}\left(a-a^{\prime}\right)+\frac{(u-a)^{2} \mathrm{~A}^{\prime}}{2} \mathrm{p}^{\prime \prime \prime}\left(a-a^{\prime}\right)+\cdots \\
& +\mathrm{A}_{1} \mathrm{p}^{\prime}\left(a-a_{1}\right)+\left(u-a_{1}\right) \mathrm{A}_{1} \mathrm{p}^{\prime \prime}\left(a-a_{1}\right)+\frac{\left(u-a_{1}\right)^{2} \mathrm{~A}_{1}}{2} \mathrm{p}^{\prime \prime \prime}\left(a-a_{1}\right)+\cdots \\
& +\mathrm{A}_{1}^{\prime} \mathrm{p}^{\prime}\left(a-a_{1}{ }^{\prime}\right)+\left(u-a_{1}^{\prime}\right) \mathrm{A}_{1}^{\prime} \mathrm{p}^{\prime \prime}\left(a-a_{1}{ }^{\prime}\right)+\frac{\left(u-a_{1}^{\prime}\right)^{2} \mathrm{~A}_{1}^{\prime}}{2} \mathrm{p}^{\prime \prime \prime}\left(a-a_{1}{ }^{\prime}\right)+\cdots \\
& =-\frac{2 \mathrm{~A}}{(u-a)^{2}}+\mathrm{A}^{\prime} \mathrm{p}^{\prime}\left(a-a^{\prime}\right)+\mathrm{A}_{1} \mathrm{p}^{\prime}\left(a-a_{1}\right)+\mathrm{A}_{1}^{\prime} \mathrm{p}^{\prime}\left(a-a_{1}^{\prime}\right) \\
& +\left\{\mathrm{A}^{\prime} \mathrm{p}^{\prime \prime}\left(a-a^{\prime}\right)+\mathrm{A}_{1} \mathrm{p}^{\prime \prime}\left(a-a_{1}\right)+\mathrm{A}_{1}^{\prime} \mathrm{p}^{\prime \prime}\left(a-a_{1}{ }^{\prime}\right)+\frac{\mathrm{A} g_{2}}{10}\right\}(u-a) \\
& +\left\{\mathrm{A}^{\prime} \mathrm{p}^{\prime \prime \prime}\left(a-a^{\prime}\right)+\mathrm{A}_{1} \mathrm{p}^{\prime \prime \prime}\left(a-a_{1}\right)+\mathrm{A}_{1}^{\prime} \mathrm{p}^{\prime \prime \prime}\left(a-a_{1}^{\prime}\right)\right\} \frac{(u-a)^{2}}{2}+\ldots \\
& \therefore\left(\frac{d x}{d u}\right)^{2}=\frac{4 \mathrm{~A}^{2}}{(u-a)^{6}}-\frac{4 \mathrm{~A}\left\{\mathrm{~A}^{\prime} \mathrm{p}^{\prime}\left(a-a^{\prime}\right)+\mathrm{A}_{1} \mathrm{p}^{\prime}\left(a-a_{1}\right)+\mathrm{A}_{1}^{\prime} \mathrm{p}^{\prime}\left(a-a_{1}^{\prime}\right)\right\}}{(u-a)^{3}} \\
& \frac{-4 \mathbf{A}\left\{\mathbf{A}^{\prime} \mathbf{p}^{\prime \prime}\left(a-a^{\prime}\right)+\mathbf{A}_{1} \mathrm{p}^{\prime \prime}\left(a-a_{1}\right)+\mathbf{A}_{1}^{\prime} \mathbf{p}^{\prime \prime}\left(a-a_{1}\right)+\frac{\mathbf{A} g_{2}}{10}\right\}}{(u-a)^{2}} \\
& \frac{-2 \mathrm{~A}\left\{\mathrm{~A}^{\prime} \mathrm{p}^{\prime \prime \prime}\left(a-a^{\prime}\right)+\mathrm{A}_{1} \mathrm{p}^{\prime \prime \prime}\left(a-a_{1}\right)+\mathrm{A}_{1}^{\prime} \mathrm{p}^{\prime \prime \prime}\left(a-a_{1}^{\prime}\right)\right\}}{u-a}+\mathrm{P}(u-a),
\end{aligned}
$$

where $\mathrm{P}(u-a)$ is finite at the point $u=a$.

Similarly, in the neighbourhood of $u=a_{1}$ we get

$$
\begin{aligned}
\left(\frac{d x}{d u}\right)^{2} & =\frac{4 \mathrm{~A}_{1}^{2}}{\left(u-a_{1}\right)^{6}}-\frac{4 \mathrm{~A}_{1}\left\{\mathrm{Ap}^{\prime}\left(a_{1}-a\right)+\mathrm{A}^{\prime} \mathrm{p}^{\prime}\left(a_{1}-a^{\prime}\right)+\mathrm{A}_{1}^{\prime} \mathrm{p}^{\prime}\left(a_{1}-a_{1}^{\prime}\right)\right\}}{\left(u-a_{1}\right)^{3}} \\
& \frac{-4 \mathrm{~A}_{1}\left\{\mathrm{Ap}^{\prime \prime}\left(a_{1}-a\right)+\mathrm{A}^{\prime} \mathbf{p}^{\prime \prime}\left(a_{1}-a^{\prime}\right)+\mathrm{A}_{1}^{\prime} \mathrm{p}^{\prime \prime}\left(a_{1}-a_{1}^{\prime}\right)+\frac{\mathrm{A}_{1} g_{2}}{10}\right\}}{\left(u-a_{1}\right)^{2}} \\
& \frac{-2 \mathrm{~A}_{1}\left\{\mathbf{A p}^{\prime \prime \prime}\left(a_{1}-a\right)+\mathrm{A}^{\prime} \mathrm{p}^{\prime \prime \prime}\left(a_{1}-a^{\prime}\right)+\mathrm{A}_{1}^{\prime} \mathrm{p}^{\prime \prime \prime}\left(a_{1}-a_{1}^{\prime}\right)\right\}}{u-a_{1}}+\mathrm{P}_{1}\left(u-a_{1}\right) .
\end{aligned}
$$

By putting $B$ for $A$ throughout, we get the corresponding two expressions for $\left(\frac{d y}{d u}\right)^{2}$. 
39

$\underset{8}{9}$

$\stackrel{\oplus}{\stackrel{8}{8}}$

弯

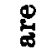

$\stackrel{9}{\$} \quad \frac{2}{7}$

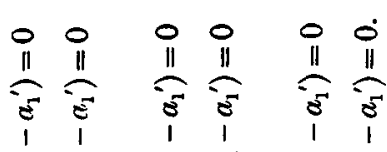

\& 8 \& 8 ह

䓵

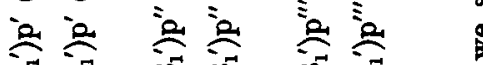

嗬

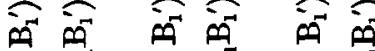

$\oplus$ की थ लि क लि

$++++$

$++$

is

$++$

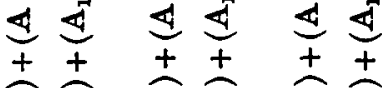

है के के के

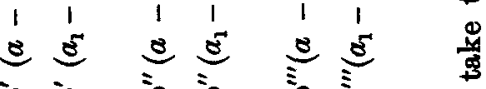

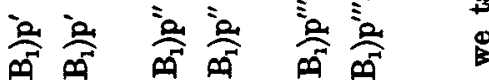

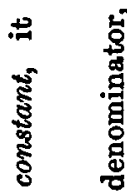

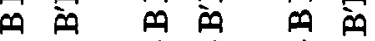

$++++++t$

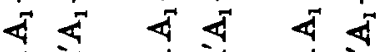

4过 4 य 4

$\frac{+}{8} \frac{t}{8} \stackrel{+}{\frac{8}{8}} \stackrel{+}{\frac{1}{8}} \frac{1}{8}$

nI

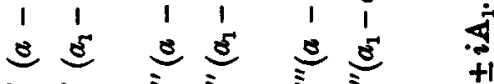

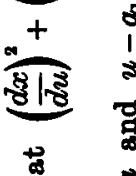

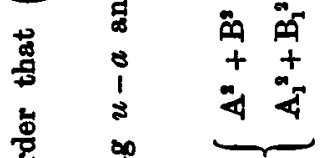

in

ic =

总

奥

商奥 商

$++++++$

过

过

过

क

苞

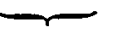

它

宣

$\vec{z}$

4

$+$

A

范

总 总

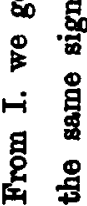


Hence we have

$$
\begin{aligned}
& \begin{cases}\mathrm{B}=i \mathrm{~A}, & \mathrm{~B}^{\prime}=-i \mathrm{~A}^{\prime} \\
\mathrm{B}_{1}=i \mathrm{~A}_{1}, & \mathrm{~B}_{1}^{\prime}=-i \mathrm{~A}_{1}^{\prime}\end{cases} \\
& \therefore \quad A A^{\prime}+B B^{\prime}=2 A A^{\prime} \\
& A A_{1}+B B_{1}=0 \\
& A A_{1}^{\prime}+B B_{1}^{\prime}=2 A A_{1}^{\prime} \\
& A^{\prime} A_{1}+B^{\prime} B_{1}=2 A^{\prime} A_{1} \\
& A_{1} A_{1}{ }^{\prime}+B_{1} B_{1}^{\prime}=2 A_{1} A_{1}^{\prime}
\end{aligned}
$$

so that the foregoing equations of condition reduce to

$$
\left.\begin{array}{l}
A^{\prime} p^{\prime}\left(a-a^{\prime}\right)+A_{1}^{\prime} p^{\prime}\left(a-a_{1}\right)=0 \\
A^{\prime} p^{\prime}\left(a_{1}-a^{\prime}\right)+A_{1}^{\prime} p^{\prime}\left(a_{1}-a_{1}\right)=0
\end{array}\right\} \text { from II. }
$$

Now these conditions will evidently be fulfilled if we can find an elliptic function which will be zero at $a$ and $a_{1}$, and infinite at $a^{\prime}$ and $a_{1}^{\prime}$. Hence the function has the form

$$
f(u)=c \mathrm{p}^{\prime}\left(u-a^{\prime}\right)+c_{1} \mathrm{p}^{\prime}\left(u-a_{1}{ }^{\prime}\right) .
$$

Also, we see from the above equations of condition that the zeros and infinities are each of the 3rd order, so that we may also represent $f(u)$ as in the equation viii. (1).

Therefore we may write

(4) $\frac{1}{\mathrm{C}} f(u)=\frac{\sigma^{3}(u-a) \sigma^{3}\left(u-a_{1}\right)}{\sigma^{3}\left(u-a^{\prime}\right) \sigma^{3}\left(u-a_{1}^{\prime}\right)}=c \mathrm{p}^{\prime}\left(u-a^{\prime}\right)+c_{1} \mathrm{p}^{\prime}\left(u-a_{1}^{\prime}\right)$

and these are the equations we have to satisfy.

If we develop $f(u)$ in the neighbourhood of $a^{\prime}$ we get,* remembering that

$$
\frac{d}{d u} \log \sigma(u-a)=\frac{\sigma^{\prime}}{\sigma}(u-a) \dagger, \text { and } \frac{d^{2}}{d u^{2}} \log \sigma(u-a)=-\mathrm{p}(u-a),
$$

* Formeln, p. 10, and footnote p. 26.

+ It ought perhaps to have been mentioned that $\frac{\sigma^{\prime}}{\sigma} u$ is a contraction for $\frac{\sigma^{\prime} u}{\sigma u}$. 
(5) $\frac{1}{\mathrm{C}} f(u)=\frac{\sigma^{3}\left(a^{\prime}-a\right) \sigma^{3}\left(a^{\prime}-a_{1}\right)}{\sigma^{3}\left(a^{\prime}-a_{1}^{\prime}\right)} \times$

$$
\begin{aligned}
{\left[\frac{1}{\left(u-a^{\prime}\right)^{2}}\right.} & +3\left\{\frac{\sigma^{\prime}}{\sigma}\left(a^{\prime}-a\right)+\frac{\sigma^{\prime}}{\sigma}\left(a^{\prime}-a_{1}\right)-\frac{\sigma^{\prime}}{\sigma}\left(a^{\prime}-a_{1}^{\prime}\right)\right\} \frac{1}{\left(u-a^{\prime}\right)^{2}} \\
+ & \left\{9\left(\frac{\sigma^{\prime}}{\sigma}\left(a^{\prime}-a\right)+\frac{\sigma^{\prime}}{\sigma}\left(a^{\prime}-a_{1}\right)-\frac{\sigma^{\prime}}{\sigma}\left(a^{\prime}-a_{2}{ }^{\prime}\right)\right)^{2}\right. \\
& \left.\left.-3\left(\mathrm{p}\left(a^{\prime}-a\right)+\mathrm{p}\left(a^{\prime}-a_{1}\right)-\mathrm{p}\left(a^{\prime}-a_{2}^{\prime}\right)\right)\right\} \frac{1}{2\left(u-a^{\prime}\right)}+\ldots\right]
\end{aligned}
$$

By putting $a_{1}^{\prime}$ instead of $a^{\prime}$ we get the corresponding expression for the development of the function in the neighbourhood of $a_{1}$.

For shortness put

(6) $\left\{\begin{array}{l}\mathrm{K} \text { for } \frac{\sigma^{3}\left(a^{\prime}-a\right) \sigma^{3}\left(a^{\prime}-a_{1}\right)}{\sigma^{3}\left(a^{\prime}-a_{1}^{\prime}\right)} \\ \mathrm{K}_{1} \text { for } \frac{\sigma^{\prime}}{\sigma}\left(a^{\prime}-a\right)+\frac{\sigma^{\prime}}{\sigma}\left(a^{\prime}-a_{1}\right)-\frac{\sigma^{\prime}}{\sigma}\left(a^{\prime}-a_{1}\right) \\ \mathrm{K}_{2} \text { for } \mathrm{p}\left(a^{\prime}-a\right)+\mathrm{p}\left(a^{\prime}-a_{1}\right)-\mathrm{p}\left(a^{\prime}-a_{2}\right)\end{array}\right.$

and let $K^{\prime}, K_{1}^{\prime}, K_{2}^{\prime}$ be the corresponding expressions when we put $a_{1}$ for $a$, and $a_{1}^{\prime}$ for $a^{\prime}$.

Remembering also that

$$
\begin{aligned}
* \mathrm{p}^{\prime}\left(u-a^{\prime}\right) & =-\frac{2}{\left(u-a^{\prime}\right)^{3}}+\ldots . . \\
\dagger \mathrm{p}\left(u-a^{\prime}\right) & =\frac{1}{\left(u-a^{\prime}\right)^{2}}+\ldots . \\
\dagger \frac{\sigma^{\prime}}{\sigma}\left(u-a^{\prime}\right) & =\frac{1}{u-a^{\prime}}+\ldots . .
\end{aligned}
$$

and that $\frac{\sigma^{\prime}}{\sigma}(-u)=-\frac{\sigma^{\prime}}{\sigma}(u)$, and $p(-u)=p u$.

we get from (4) and (5),

$$
\begin{array}{r}
\text { (7) } \frac{1}{\mathrm{C}} f(u)=\frac{\sigma^{3}(u-a) \sigma^{3}\left(u-a_{2}\right)}{\sigma^{3}\left(u-a^{\prime}\right) \sigma^{2}\left(u-a_{1}^{\prime}\right)}=-\frac{\mathrm{K}}{2} \mathrm{p}^{\prime}\left(u-a^{\prime}\right)-\frac{\mathrm{K}^{\prime}}{2} \mathrm{p}^{\prime}\left(u-a_{1}{ }^{\prime}\right) \\
+3 \mathrm{KK}_{1} \mathrm{p}\left(u-a^{\prime}\right)+3 \mathrm{~K}^{\prime} \mathrm{K}_{1}^{\prime} \mathrm{p}\left(u-a_{1}{ }^{\prime}\right)+\frac{1}{2} \mathrm{~K}\left(9 \mathrm{~K}_{1}^{2}-3 \mathrm{~K}_{2}\right) \frac{\sigma^{\prime}}{\sigma}\left(u-a^{\prime}\right) \\
+\frac{1}{2} \mathrm{~K}^{\prime}\left(9 \mathrm{~K}_{1}^{\prime}-3 \mathrm{~K}_{2}^{\prime}\right) \frac{\sigma^{\prime}}{\sigma}\left(u-a_{1}^{\prime}\right)+\text { constant. }
\end{array}
$$

- Formeln, p. 11.

† Formeln, p. 10. 
By putting $u=0$ on both sides we find that the

(8) Constant $=\frac{\sigma^{3}(a) \sigma^{3}\left(a_{1}\right)}{\sigma^{3}\left(a^{\prime}\right) \sigma^{3}\left(a_{1}^{\prime}\right)}-\frac{\mathrm{K}}{2} \mathrm{p}^{\prime}\left(a^{\prime}\right)-\frac{\mathrm{K}^{\prime}}{2} \mathrm{p}^{\prime}\left(a_{1}{ }^{\prime}\right)-3 \mathrm{KK}_{1} \mathrm{p}\left(a^{\prime}\right)$

$$
-3 K^{\prime} K_{1}^{\prime} p\left(a_{1}\right)+\frac{K}{2}\left(9 K_{1}{ }^{2}-3 K_{2}\right) \frac{\sigma^{\prime}}{\sigma}\left(a^{\prime}\right)+\frac{K^{\prime}}{2}\left(9 K_{1}{ }^{\prime 2}-3 K_{2}{ }^{\prime}\right) \frac{\sigma^{\prime}}{\sigma}\left(a_{1}{ }^{\prime}\right) \text {. }
$$

We see from (7) that the necessary and sufficient conditions that $f(u)$ take the second form required in (4) are that $\mathbf{K}_{1}, \mathbf{K}_{1}^{\prime}, \mathbf{K}_{2}, \mathbf{K}_{2}^{\prime}$ as well as the constant term vanish, for then (7) will become

$$
\text { (7a) } \frac{1}{\mathrm{C}} f(u)=\frac{\sigma^{3}(u-a) \sigma^{3}\left(u-a_{1}\right)}{\sigma^{3}\left(u-a^{\prime}\right) \sigma^{3}\left(u-a_{1}^{\prime}\right)}=-\frac{\mathrm{K}}{2} \mathrm{p}^{\prime}\left(u-a^{\prime}\right)-\frac{\mathrm{K}^{\prime}}{2} \mathrm{p}^{\prime}\left(u-a_{1}^{\prime}\right) .
$$

But the sum of the zeros equals the sum of the infinities [see VIII. (2)].

$$
\begin{aligned}
& \left.\begin{array}{l}
\text { i.e. } a+a_{1}=a^{\prime}+a_{1}^{\prime} \\
\therefore a^{\prime}-a=-\left(a_{1}^{\prime}-a_{1}\right)
\end{array}\right\} \therefore \text { from (6) } \\
& \text { and } \left.a^{\prime}-a_{1}=-\left(a_{1}^{\prime}-a\right)\right\} \mathrm{K}=-\mathbf{K}^{\prime}, \mathrm{K}_{1}=-\mathbf{K}_{1}^{\prime}, \mathbf{K}_{2}=\mathbf{K}_{2}^{\prime} \text {. }
\end{aligned}
$$

and of course $a^{\prime}-a_{1}^{\prime}=-\left(a_{1}^{\prime}-a^{\prime}\right)$

Wherefore the conditions that $\mathrm{K}_{1}, \mathrm{~K}_{1}^{\prime}, \mathrm{K}_{2}, \mathrm{~K}_{2}^{\prime}$, and the constant in (7) vanish are equivalent as we see from (6) to finding the conditions that

$$
\frac{\sigma^{\prime}}{\sigma}\left(a^{\prime}-a\right)+\frac{\sigma^{\prime}}{\sigma}\left(a^{\prime}-a_{1}\right)-\frac{\sigma^{\prime}}{\sigma}\left(a^{\prime}-a_{1}^{\prime}\right)=0
$$

and (10)

$$
\mathrm{p}\left(a^{\prime}-a\right)+\mathrm{p}\left(a^{\prime}-a_{1}\right)-\mathrm{p}\left(a^{\prime}-a_{1}^{\prime}\right)=0
$$

and also constant $=0$.

But since $a^{\prime}-a_{1}^{\prime}=\left(a^{\prime}-a\right)+\left(a^{\prime}-a_{1}\right)$; then applying the two addition formulae.

$$
\begin{gathered}
\frac{\sigma^{\prime}}{\sigma}(u+v)=\frac{\sigma^{\prime}}{\sigma}(u)+\frac{\sigma^{\prime}}{\sigma}(v)+\frac{1}{2} \frac{\mathrm{p}^{\prime} u-\mathrm{p}^{\prime} v}{\mathrm{p} u-\mathrm{p} v} * \\
\mathrm{p}(u+v)=\frac{1}{2}\left(\frac{\mathrm{p}^{\prime} u-\mathrm{p}^{\prime} v}{\mathrm{p} u-\mathrm{p} v}\right)^{2}-\mathrm{p} u-\mathrm{p} v \dagger
\end{gathered}
$$

* Formeln, p. 13.

† Formeln, p. 14. 
(9) and (10) are reduced respectively to finding the conditions that

$$
\begin{aligned}
& \frac{\mathrm{p}^{\prime}\left(a^{\prime}-a\right)-\mathrm{p}^{\prime}\left(a^{\prime}-a_{1}\right)}{\mathrm{p}\left(a^{\prime}-a\right)-\mathrm{p}\left(a^{\prime}-a_{1}\right)}=0 \\
& \mathrm{p}\left(a^{\prime}-a\right)+\mathrm{p}\left(a^{\prime}-a_{1}\right)=0 .
\end{aligned}
$$

But, as already stated,

$$
\begin{array}{lll}
\mathrm{p}^{\prime} \omega=0, & \mathrm{p}^{\prime} \omega^{\prime}=0, & \mathrm{p}^{\prime} \omega^{\prime \prime}=0 \\
\mathrm{p} \omega=e_{1}, & \mathrm{p} \omega^{\prime}=e_{3}, & \mathrm{p} \omega^{\prime \prime}=e_{2} .
\end{array}
$$

We see, therefore, that (11) will be satisfied if $a^{\prime}-a, a^{\prime}-a_{1}$ are semiperiods; and, since $e_{1}+e_{2}+e_{3}=0$ (see p. 26), we see that (12) will also be satisfied if one of the $e$ 's vanishes. To find which $e$ can vanish, we proceed thus :

$k$, the modulus of the elliptic function, must be $+{ }^{* 0}$ and $<1$, and

$$
k^{2}=\frac{e_{2}-e_{3}}{e_{1}-e_{3}} \cdot *
$$

If $e_{1}=0$, then $k^{2}=2$; if $e_{2}=0$, then $k^{2}=\frac{1}{2}$; if $e_{3}=0$, then $k^{2}=-1$; so that we can only possibly have $e_{3}=0$.

$$
a^{\prime}-a=\omega^{\prime} ; a^{\prime}-a_{1}=\omega ; a^{\prime}-a_{1}^{\prime}=\left(a^{\prime}-a_{1}\right)+\left(a^{\prime}-a\right)=\omega+\omega^{\prime}=\omega^{\prime \prime} .
$$

Putting these values in (6) we get

$$
\mathbf{K}=\frac{\sigma^{3}\left(\omega^{\prime}\right) \sigma^{3}(\omega)}{\sigma^{3}\left(\omega^{\prime \prime}\right)} \text {; and, as we have already seen, } K^{\prime}=-\mathbf{K} \text {. }
$$

With these data it can now be shown that the constant (8) vanishes, and therefore the proof is complete that the necessary conditions can be fulfilled that the third member of (7) is reducible to the form of the third member of (4), which in turn we have shown to be the condition that the curve is algebraic.

To find the cartesian equation of the curve we may proceed thus :

Insert the above values of $K$ and $K^{\prime}$ in $(7 a)$, and at the same time put

$$
\frac{1}{0}=-\frac{\sigma^{3}(\omega) \sigma^{3}\left(\omega^{\prime}\right)}{2 \sigma^{3}\left(\omega^{\prime \prime}\right)}
$$

* Formeln, p. 30. 
we then get

$$
f(u)=\mathrm{p}^{\prime}\left(u-a^{\prime}\right)-\mathrm{p}^{\prime}\left(u-a_{1}{ }^{\prime}\right) .
$$

Comparing this with our original equations (1) and (2) we see that

$$
\mathbf{A}^{\prime}=1, \quad \mathbf{A}_{1}^{\prime}=-1 \text {. }
$$

Also, according to (13) we can put*

$$
\begin{cases}a=\frac{\omega}{2}-\frac{\omega^{\prime}}{2}, & a_{1}=-\frac{\omega}{2}+\frac{\omega^{\prime}}{2} \\ a^{\prime}=\frac{\omega}{2}+\frac{\omega^{\prime}}{2}, & a_{1}^{\prime}=-\frac{\omega}{2}-\frac{\omega^{\prime}}{2} .\end{cases}
$$

Hence in (1) we have, remembering also the values in (3),

$$
\begin{aligned}
& x=\mathrm{p}\left(u-\frac{\omega}{2}+\frac{\omega^{\prime}}{2}\right)+\mathrm{p}\left(u-\frac{\omega}{2}-\frac{\omega^{\prime}}{2}\right)-\mathrm{p}\left(u+\frac{\omega}{2}-\frac{\omega^{\prime}}{2}\right)-\mathrm{p}\left(u+\frac{\omega}{2}+\frac{\omega^{\prime}}{2}\right) \\
& y=i \mathrm{p}\left(u-\frac{\omega}{2}+\frac{\omega^{\prime}}{2}\right)-i \mathrm{p}\left(u-\frac{\omega}{2}-\frac{\omega^{\prime}}{2}\right)-i \mathrm{p}\left(u+\frac{\omega^{\prime}}{2}-\frac{\omega^{\prime}}{2}\right)+i \mathrm{p}\left(u+\frac{\omega^{\prime}}{2}+\frac{\omega^{\prime}}{2}\right) .
\end{aligned}
$$

From this we get

$$
\begin{aligned}
& x+i y=2\left[\mathrm{p}\left(u-\frac{\omega}{2}-\frac{\omega^{\prime}}{2}\right)-\mathrm{p}\left(u+\frac{\omega}{2}+\frac{\omega^{\prime}}{2}\right)\right] \\
& x-i y=2\left[\mathrm{p}\left(u-\frac{\omega^{\prime}}{2}+\frac{\omega^{\prime}}{2}\right)-\mathrm{p}\left(u+\frac{\omega^{\prime}}{2}-\frac{\omega^{\prime}}{2}\right)\right]
\end{aligned}
$$

But $\mathrm{p}\left(u-\frac{\omega}{2}-\frac{\omega^{\prime}}{2}\right)=\mathrm{p}\left(u-\frac{\omega^{\prime \prime}}{2}\right)$ and $\mathrm{p}\left(u+\frac{\omega}{2}+\frac{\omega^{\prime}}{2}\right)=\mathrm{p}\left(u+\frac{\omega^{\prime \prime}}{2}\right)$

Also (see Formeln, p. 14).

(17) $\mathrm{p}(u+v) \cdot \mathrm{p}(u-v)=\frac{\left(\mathrm{p} u \mathrm{p} v+\frac{1}{4} g_{2}\right)^{2}+g_{3}(\mathrm{p} u+\mathrm{p} v)}{(\mathrm{p} u-\mathrm{p} v)^{2}}$

Remembering that $e_{2}=0$

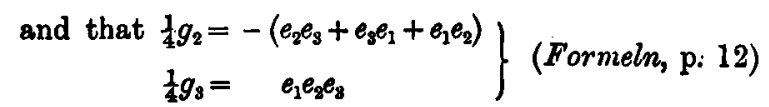

$\therefore$ in this case $\quad \frac{1}{4} g_{2}=e_{8}^{2}$, and $g_{3}=0$.

Wherefore (17) gives us

$$
\mathrm{p}\left(u+\frac{\omega^{\prime \prime}}{2}\right) \cdot \mathrm{p}\left(u-\frac{\omega^{\prime \prime}}{2}\right)=\frac{\left(\mathrm{p} u \mathrm{p} \frac{\omega^{\prime \prime}}{2}+e_{\mathrm{s}}^{2}\right)^{2}}{\left(\mathrm{p} u-\mathrm{p} \frac{\omega^{\prime \prime}}{2}\right)^{2}}
$$

- Compare these values with Kiepert's values on p. 32 for the group of curves which includes the lemniscate. 
Again (see Formeln, p. 14)

$$
\begin{gathered}
\frac{\left(\mathrm{p}^{2} u+\frac{1}{4} g_{2}\right)^{2}+2 g_{3} \mathrm{p} u}{4 \mathrm{p}^{3} u-g_{2} \mathrm{p} u-g_{3}}=\mathrm{p}(2 u) \\
\text { Therefore, } \quad \frac{\left\{\mathrm{p}^{2}\left(\frac{\omega^{\prime \prime}}{2}\right)+e_{3}^{2}\right\}^{2}}{\text { etc. }}=\mathrm{p} \omega^{\prime \prime}=e_{2}=0 \\
\text { whence } \frac{\omega^{\prime \prime}}{2}=i e_{3} .
\end{gathered}
$$

Therefore (18) gives

$$
\begin{gathered}
\mathrm{p}\left(u+\frac{\omega^{\prime \prime}}{2}\right) \cdot \mathrm{p}\left(u-\frac{\omega^{\prime \prime}}{2}\right)=\left(\frac{\mathrm{p} u \cdot i e_{3}+e_{3}{ }^{2}}{\mathrm{p} u-i e_{3}}\right)^{2}=-e_{3}{ }^{2} \\
\therefore \mathrm{p}\left(u+\frac{\omega^{\prime \prime}}{2}\right) \text { or } \mathrm{p}\left(u+\frac{\omega}{2}+\frac{\omega^{\prime}}{2}\right)=\frac{-e_{8}{ }^{2}}{\mathrm{p}\left(u-\frac{\omega}{2}-\frac{\omega^{\prime}}{2}\right)}
\end{gathered}
$$

Similarly $\mathrm{p}\left(u-\frac{\omega}{2}+\frac{\omega^{\prime}}{2}\right)=e_{3}+\frac{2 e_{3}^{3}}{\mathrm{p}\left(u-\frac{\omega}{2}-\frac{\omega^{\prime}}{2}\right)-e_{3}}$

and

$$
\mathrm{p}\left(u+\frac{\omega}{2}-\frac{\omega^{\prime}}{2}\right)=e_{3}-\frac{2 e_{3} \mathrm{p}\left(u-\frac{\omega}{2}-\frac{\omega^{\prime}}{2}\right)}{e_{3}+\mathrm{p}\left(u-\frac{\omega}{2}-\frac{\omega^{\prime}}{2}\right)}
$$

Putting these values in (15) and (16) they reduce to

$$
\begin{aligned}
& x+i y=2 \frac{\mathrm{p}^{2}\left(u-\frac{\omega}{2}-\frac{\omega^{\prime}}{2}\right)+e_{3}^{2}}{\mathrm{p}\left(u-\frac{\omega}{2}-\frac{\omega^{\prime}}{2}\right)} \\
& x-i y=4 e_{3} \frac{\mathrm{p}^{2}\left(u-\frac{\omega}{2}-\frac{\omega^{\prime}}{2}\right)+e_{3}^{2}}{\mathrm{p}^{2}\left(u-\frac{\omega}{2}-\frac{\omega^{\prime}}{2}\right)-e_{8}^{2}} .
\end{aligned}
$$

Eliminating the $\mathrm{p}$ - function between these, we get finally

$$
\left(x^{2}+y^{2}\right)^{2}=32 e_{3}^{2}\left(x^{2}-y^{2}\right)
$$

i.e., the curve is a lemniscate. 
$\mathbf{x}$

As we have already said, the great advantage of Lilienthal's notation is that it can be readily extended to space curves. To show this Lilienthal takes the case analogous to the plane lemniscate. We have

$$
\left\{\begin{array}{l}
x=\mathrm{Ap}(u-a)+\mathrm{A}^{\prime} \mathrm{p}\left(u-a^{\prime}\right)+\mathrm{A}_{1} \mathrm{p}\left(u-a_{1}\right)+\mathrm{A}_{1}^{\prime} \mathrm{p}\left(u-a_{1}{ }^{\prime}\right) \\
y=\mathrm{Bp}(u-a)+\mathrm{B}^{\prime} \mathrm{p}\left(u-a^{\prime}\right)+\mathrm{B}_{1} \mathrm{p}\left(u-a_{1}\right)+\mathrm{B}_{1}^{\prime} \mathrm{p}\left(u-a_{1}{ }^{\prime}\right) \\
z=\mathrm{C} \mathrm{p}(u-a)+\mathrm{C}^{\prime} \mathrm{p}\left(u-a^{\prime}\right)+\mathrm{C}_{1} \mathrm{p}\left(u-a_{1}\right)+\mathrm{C}_{1}^{\prime} \mathrm{p}\left(u-a_{1}{ }^{\prime}\right)
\end{array}\right.
$$

In order that $\left(\frac{d x}{d u}\right)^{2}+\left(\frac{d y}{d u}\right)^{2}+\left(\frac{d z}{d u}\right)^{2}$ have no point $x^{2}$ infinity we have (see p. 39)

$$
\begin{aligned}
& \text { I. }\left\{\begin{array}{l}
A^{2}+B^{2}+C^{2}=0 \\
A_{1}{ }^{2}+B_{1}{ }^{2}+C_{1}{ }^{2}=0
\end{array}\right. \\
& \text { II. }\left\{\begin{array}{r}
\left(\mathrm{AA}^{\prime}+\mathrm{BB}^{\prime}+\mathrm{CC}^{\prime}\right) \mathrm{p}^{\prime}\left(a-a^{\prime}\right)+\left(\mathrm{A}_{1}+\mathrm{B} \mathrm{B}_{1}+\mathrm{CC}_{1}\right) \mathrm{p}^{\prime}\left(a-a_{1}\right) \\
+\left(\mathrm{AA}_{1}^{\prime}+\mathrm{B} \mathrm{B}_{1}^{\prime}+\mathrm{CC}_{1}^{\prime}\right) \mathrm{p}^{\prime}\left(a-a_{1}^{\prime}\right)=0 \\
\left(\mathrm{AA}_{1}+\mathrm{BB}_{1}+\mathrm{CC}_{1}\right) \mathrm{p}^{\prime}\left(a_{1}-a\right)+\left(\mathrm{A}^{\prime} \mathrm{A}_{1}+\mathrm{B}^{\prime} \mathrm{B}_{1}+\mathrm{C}^{\prime} \mathrm{C}_{1}\right) \mathrm{p}^{\prime}\left(a_{1}-a^{\prime}\right) \\
+\left(\mathrm{A}_{1}^{\prime} \mathrm{A}_{1}+\mathrm{B}_{1}^{\prime} \mathrm{B}_{1}+\mathrm{C}_{1}^{\prime} \mathrm{C}_{1}\right) \mathrm{p}^{\prime}\left(a_{1}-a_{1}^{\prime}\right)=0
\end{array}\right.
\end{aligned}
$$

and systems corresponding to III. and IV. by putting $\mathrm{p}^{\prime \prime}$ and $\mathrm{p}^{\prime \prime \prime}$ instead of $\mathrm{p}^{\prime}$ in II.

These equations, as we have seen, assume the simplest form when $a, a^{\prime}, a_{1}$, and $a_{1}{ }^{\prime}$ have the values given in IX. (14); so that

$$
\begin{aligned}
& a-a^{\prime}=-\left(a_{1}-a_{1}{ }^{\prime}\right)=-\omega^{\prime}=\omega^{\prime} \text { (since } 2 \omega^{\prime} \text { is a period) } \\
& a-a_{1}=\omega-\omega^{\prime}=\omega+\omega^{\prime}=\omega^{\prime \prime} \\
& a-a_{1}^{\prime}=-\left(a_{1}-a^{\prime}\right)=\omega .
\end{aligned}
$$

But $p^{\prime} \omega, p^{\prime} \omega^{\prime}$, and $p^{\prime} \omega^{\prime \prime}$ are zero, therefore also $p^{\prime \prime \prime}$ vanishes for the semiperiods, ${ }^{*}$ so that the conditions II. and IV. are at once satisfied, and we are left to find the necessary and sufficient conditions that

I. $\left\{\begin{array}{l}A^{2}+B^{2}+C^{2}=0 \\ A_{1}{ }^{2}+B_{1}^{2}+C_{1}^{2}=0\end{array}\right.$

* Halphen's F. E., p. 27. 
III. $\left\{\begin{array}{c}\left(A A^{\prime}+B B^{\prime}+C C^{\prime}\right) p^{\prime \prime} \omega^{\prime}+\left(A A_{1}+B B_{1}+C C_{1}\right) p^{\prime \prime} \omega^{\prime \prime} \\ +\left(A A_{1}^{\prime}+B B_{1}{ }^{\prime}+C C_{1}^{\prime}\right) p^{\prime \prime} \omega=0 \\ \left(A A_{1}+B B_{1}+C C_{1}\right) p^{\prime \prime} \omega^{\prime \prime}+\left(A^{\prime} A_{1}+B^{\prime} B_{1}+C^{\prime} C_{1}\right) p^{\prime \prime} \omega \\ +\left(A_{1}^{\prime} A_{1}+B_{1}^{\prime} B_{1}+C_{1} C_{1}\right) p^{\prime \prime} \omega^{\prime}=0\end{array}\right.$

Let us now put
$A=r e^{i a}$,
$\mathbf{B}=\rho e^{i \beta}$,
$\mathbf{C}=\mathbf{R} e^{i \gamma}$,
$\mathbf{A}^{\prime}=r e^{-i \alpha}$, etc.
$A_{1}=r_{1} e^{i a_{1}}$,
$\mathrm{B}_{1}=\rho_{1} e^{i \beta_{1}}$
$\mathrm{C}_{1}=\mathrm{R}_{1} e^{i \gamma_{1}}$,
$\mathbf{A}_{1}^{\prime}=r_{1} e^{-i a_{1}}$, etc.

so that I. becomes, remembering that $e^{2 i \alpha}=\cos 2 \alpha+i \sin 2 \alpha$,

$$
\begin{aligned}
& \sum r^{2}(\cos 2 \alpha+i \sin 2 \alpha)=0 \\
& \sum r_{1}^{2}\left(\cos 2 \alpha_{1}+i \sin 2 \alpha_{1}\right)=0
\end{aligned}
$$

or, separating the real and imaginary parts,

III. becomes

$$
I^{a} .\left\{\begin{array}{l}
\Sigma r^{2} \cos 2 a=0 \\
\Sigma r^{2} \sin 2 a=0 \\
\Sigma r_{1}{ }^{2} \cos 2 a_{1}=0 \\
\Sigma r_{1}{ }^{2} \sin 2 a_{1}=0 .
\end{array}\right.
$$

$$
\mathrm{p}^{\prime \prime} \omega^{\prime}\left(r^{2}+\rho^{2}+\mathrm{R}^{2}\right)+\mathrm{p}^{\prime \prime} \omega^{\prime \prime} \Sigma r r_{1} e^{i a+i a_{1}}+\mathrm{p}^{\prime \prime} \omega \Sigma r r_{1} e^{i a-i a_{1}}=0
$$

and another similar expression.

But

$$
\begin{aligned}
e^{i a+i a_{1}} & =(\cos a+i \sin a)\left(\cos a_{1}+i \sin a_{1}\right) \\
& =\cos \left(\alpha+a_{1}\right)+i \sin \left(\alpha+a_{1}\right)
\end{aligned}
$$

Similarly $\quad e^{i a-i a_{1}}=\cos \left(a-a_{1}\right)+i \sin \left(a-a_{1}\right)$.

Hence, putting these values in the above and separating the real and imaginary parts we get

$$
\left\{\begin{array}{l}
\mathrm{p}^{\prime \prime} \omega^{\prime} \Sigma r^{2}+\mathrm{p}^{\prime \prime} \omega^{\prime \prime} \Sigma r r_{1} \cos \left(a+a_{1}\right)+\mathrm{p}^{\prime \prime} \omega \Sigma r r_{1} \cos \left(\alpha-a_{1}\right)=0 \\
\mathrm{p}^{\prime \prime} \omega^{\prime \prime} \Sigma r r_{1} \sin \left(\alpha+a_{1}\right)+\mathrm{p}^{\prime \prime} \omega \Sigma r r_{1} \sin \left(a-a_{1}\right)=0 \\
\mathrm{p}^{\prime \prime} \omega^{\prime} \Sigma r_{1}^{2}+\mathrm{p}^{\prime \prime} \omega^{\prime \prime} \Sigma r r_{1} \cos \left(\alpha+a_{1}\right)+\mathrm{p}^{\prime \prime} \omega \Sigma r r_{1} \cos \left(\alpha-a_{1}\right)=0 \\
\mathrm{p}^{\prime \prime} \omega^{\prime \prime} \Sigma r r_{1} \sin \left(\alpha+a_{1}\right)+\mathrm{p}^{\prime \prime} \omega \Sigma r r_{1} \sin \left(a_{1}-a\right)=0
\end{array}\right.
$$


By comparing these four equations we see that we can replace them by the following :-

$$
\text { III }{ }^{a} .\left\{\begin{array}{l}
\mathrm{p}^{\prime \prime} \omega^{\prime} \Sigma r^{2}+\mathrm{p}^{\prime \prime} \omega^{\prime \prime} \Sigma r_{1} \cos \left(\alpha+a_{2}\right)+\mathrm{p}^{\prime \prime} \omega \Sigma r r_{1} \cos \left(a-a_{1}\right)=0 \\
\Sigma r^{2}=\Sigma r_{1}^{2} \\
\Sigma r_{1} \sin \alpha \cos \alpha_{1}=0 \\
\Sigma r r_{1} \cos \alpha \sin \alpha_{1}=0 .
\end{array}\right.
$$

$\mathrm{I}^{a}$. and III ${ }^{\text {a }}$. contain eight equations of condition among twelve unknowns, but one of the quantities $r, \rho, \mathbf{R}, r_{1}, \rho_{1}, \mathbf{R}_{1}$ we can from the beginning assume equal to any quantity we choose, and the three remaining andetermined quantities only refer to the rotating of the original coordinate system, so that we have sufficient data to satisfy the necessary conditions for the existence of a curve of double curvature corresponding to the plane lemniscate.

Lilienthal goes through a long analysis in order to obtain the equation of the spherical lemniscate from the equations of condition $I^{a}$. and III ${ }^{a}$. He finally obtains

$$
\begin{aligned}
& x=\operatorname{Csin} \phi \times \\
& {\left[\mathrm{p}\left(u-\frac{\omega}{2}+\frac{\omega^{\prime}}{2}\right)+\mathrm{p}\left(u-\frac{\omega}{2}-\frac{\omega^{\prime}}{2}\right)-\mathrm{p}\left(u+\frac{\omega}{2}-\frac{\omega^{\prime}}{2}\right)-\mathrm{p}\left(u+\frac{\omega}{2}+\frac{\omega^{\prime}}{2}\right)\right]} \\
& y=\operatorname{Ccos} \phi \times \\
& {\left[\mathrm{p}\left(u-\frac{\omega}{2}+\frac{\omega^{\prime}}{2}\right)+\mathrm{p}\left(u-\frac{\omega}{2}-\frac{\omega^{\prime}}{2}\right)+\mathrm{p}\left(u+\frac{\omega}{2}-\frac{\omega^{\prime}}{2}\right)+\mathrm{p}\left(u+\frac{\omega}{2}+\frac{\omega^{\prime}}{2}\right)\right]} \\
& z=i \mathrm{C} \times \\
& {\left[\mathrm{p}\left(u-\frac{\omega}{2}+\frac{\omega^{\prime}}{2}\right)-\mathrm{p}\left(u-\frac{\omega}{2}-\frac{\omega^{\prime}}{2}\right)-\mathrm{p}\left(u+\frac{\omega}{2}-\frac{\omega^{\prime}}{2}\right)+\mathrm{p}\left(u+\frac{\omega}{2}+\frac{\omega^{\prime}}{2}\right)\right]}
\end{aligned}
$$

where $O$ is a constant, and $\sin \phi=\frac{k^{2}}{1-k^{2}}, k$ being the modulus of the elliptic function.

He proves that this curve lies on the sphere*

* Lilienthal does not remark that if $\phi=\frac{\pi}{2}$, then the radius of this sphere is infinite and the above curve becomes a plane lemniscate, for it modulus is $\frac{1}{\sqrt{ } 2}\left(\right.$ since $\left.\sin \phi=\frac{k^{2}}{1-k^{2}}\right)$, and the above equations for $x$ and $z(y$ of course vanishes) are the same as those for the plane lemniscate on p. 44. See also first footnote on p. 14. 


$$
x^{2}+\left(y-2 \mathrm{C} \frac{e_{3} \sin ^{2} \phi-e_{1}}{\cos \phi}\right)^{2}+z^{2}=\frac{4 \mathrm{C}^{2}\left\{e_{2}-e_{3}+\left(e_{1}-e_{2}\right) \sin ^{2} \phi\right\}^{2}}{\cos ^{2} \phi}
$$

and on the hyperbolic cylinder

$$
\frac{\left(y+2 e_{3} \mathrm{C} \cos \phi\right)^{2}}{4 \mathrm{C}^{2} \cos ^{2} \phi\left(e_{1}-e_{2}\right)^{2}}-\frac{x^{2}}{4 \mathrm{C}^{2} \sin ^{2} \phi\left(e_{1}-e_{2}\right)^{2}}=1,
$$

and on the elliptic cylinder

$$
\frac{z^{2}}{4 \mathrm{C}^{2}\left(e_{2}-e_{3}\right)^{2}}+\frac{\left(y+2 e_{1} \mathrm{C} \cos \phi\right)^{2}}{4 \mathrm{C}^{2}\left(e_{2}-e_{3}\right)^{2} \cos ^{2} \phi}=1
$$

and that the two cylinders touch the sphere from within.

Kiepert had in his Dissertation arrived at exactly similar results. 
XI.

Although Lilienthal devoted a long chapter to a discussion of the application of his method to Kiepert's curve $r^{3}=\cos 3 \phi$ (vide. p. 36) he does not mention that there is an analagous space curve. Modifying somewhat his equations for the plane curve, let

$$
\left\{\begin{array}{r}
x=\mathrm{A} \frac{\sigma^{\prime}}{\sigma}(u-a)+\mathrm{A}^{\prime} \frac{\sigma^{\prime}}{\sigma}\left(u-a^{\prime}\right)+\mathrm{A}_{1} \frac{\sigma^{\prime}}{\sigma}\left(u-a_{1}\right)+\mathrm{A}_{1} \frac{\sigma^{\prime}}{\sigma}\left(u-a_{1}{ }^{\prime}\right) \\
+\mathrm{A}_{2} \frac{\sigma^{\prime}}{\sigma}\left(u-a_{2}\right)+\mathrm{A}_{2} \frac{\sigma^{\prime}}{\sigma}\left(u-a_{2}{ }^{\prime}\right) \\
y=\mathrm{B} \frac{\sigma^{\prime}}{\sigma}(u-a)+\mathrm{B}^{\prime} \frac{\sigma^{\prime}}{\sigma}\left(u-a^{\prime}\right)+\mathrm{B}_{1} \frac{\sigma^{\prime}}{\sigma}\left(u-a_{1}\right)+\mathrm{B}_{1}, \frac{\sigma^{\prime}}{\sigma}\left(u-a_{1}\right) \\
+\mathrm{B}_{2} \frac{\sigma^{\prime}}{\sigma}\left(u-a_{2}\right)+\mathrm{B}_{2}, \frac{\sigma^{\prime}}{\sigma}\left(u-a_{2}{ }^{\prime}\right) \\
z=\mathrm{C} \frac{\sigma^{\prime}}{\sigma}(u-a)+\mathrm{C}^{\prime} \frac{\sigma^{\prime}}{\sigma}\left(u-a^{\prime}\right)+\mathrm{C}_{1} \frac{\sigma^{\prime}}{\sigma}\left(u-a_{1}\right)+\mathrm{C}_{1}, \frac{\sigma^{\prime}}{\sigma}\left(u-a_{1}{ }^{\prime}\right) \\
+\mathrm{C}_{2} \frac{\sigma^{\prime}}{\sigma}\left(u-a_{2}\right)+\mathrm{C}_{2}, \frac{\sigma^{\prime}}{\sigma}\left(u-a_{2}{ }^{\prime}\right)
\end{array}\right.
$$

where the accented quantities are the conjugates of the corresponding unaccented ones, and

$$
\begin{array}{ll}
a=\frac{2 \omega}{3}, & a_{1}=-\frac{2 \omega}{3}+\frac{2 \omega^{\prime}}{3}, a_{2}=-\frac{2 \omega^{\prime}}{3} \\
a^{\prime}=\frac{2 \omega^{\prime}}{3}, & a_{1}^{\prime}=-\frac{2 \omega^{\prime}}{3}+\frac{2 \omega}{3}, \quad a_{2}^{\prime}=-\frac{2 \omega}{3} .
\end{array}
$$

Therefore, since $\frac{d}{d u} \frac{\sigma^{\prime}}{\sigma}(u)=\frac{d^{2}}{d u^{2}} \log \sigma u=-\mathrm{p} u$, we get

$$
\begin{array}{r}
\frac{d x}{d u}=-\mathrm{Ap}(u-a)-\mathrm{A}^{\prime} \mathrm{p}\left(u-a^{\prime}\right)-\mathrm{A}_{1} \mathrm{p}\left(u-a_{1}\right)-\mathrm{A}_{1}^{\prime} \mathrm{p}\left(u-a_{1}^{\prime}\right) \\
-\mathbf{A}_{2} \mathrm{p}\left(u-a_{2}\right)-\mathbf{A}_{2}^{\prime} \mathrm{p}\left(u-a_{2}^{\prime}\right) .
\end{array}
$$

If we develop this in the neighbourhood of $u=a$ we get (see Formeln p. 10, and footnote p. 37).

$$
\begin{aligned}
\frac{d x}{d u}=-\frac{\mathrm{A}}{(u-a)^{2}}-\ldots & -\mathrm{A}^{\prime} \mathrm{p}\left(a-a^{\prime}\right)-(u-a) \mathrm{A}^{\prime} \mathrm{p}^{\prime}\left(a-a^{\prime}\right)-\ldots \\
& -\mathrm{A}_{1} \mathrm{p}\left(a-a_{1}\right)-(u-a) \mathrm{A}_{1} \mathrm{p}^{\prime}\left(a-a_{1}\right)-\ldots \\
& -\mathbf{A}_{1}^{\prime} \mathrm{p}\left(a-a_{1}^{\prime}\right)-(u-a) \mathrm{A}_{1}^{\prime} \mathrm{p}^{\prime}\left(a-a_{1}^{\prime}\right)-\ldots \\
& -\mathrm{A}_{2} \mathrm{p}\left(a-a_{2}\right)-(u-a) \mathbf{A}_{2} \mathrm{p}^{\prime}\left(a-a_{2}\right)-\ldots \\
& -\mathbf{A}_{2}^{\prime} \mathrm{p}\left(a-a_{2}^{\prime}\right)-(u-a) \mathbf{A}_{2}^{\prime} \mathrm{p}^{\prime}\left(a-a_{2}^{\prime}\right)-\ldots
\end{aligned}
$$




\section{1}

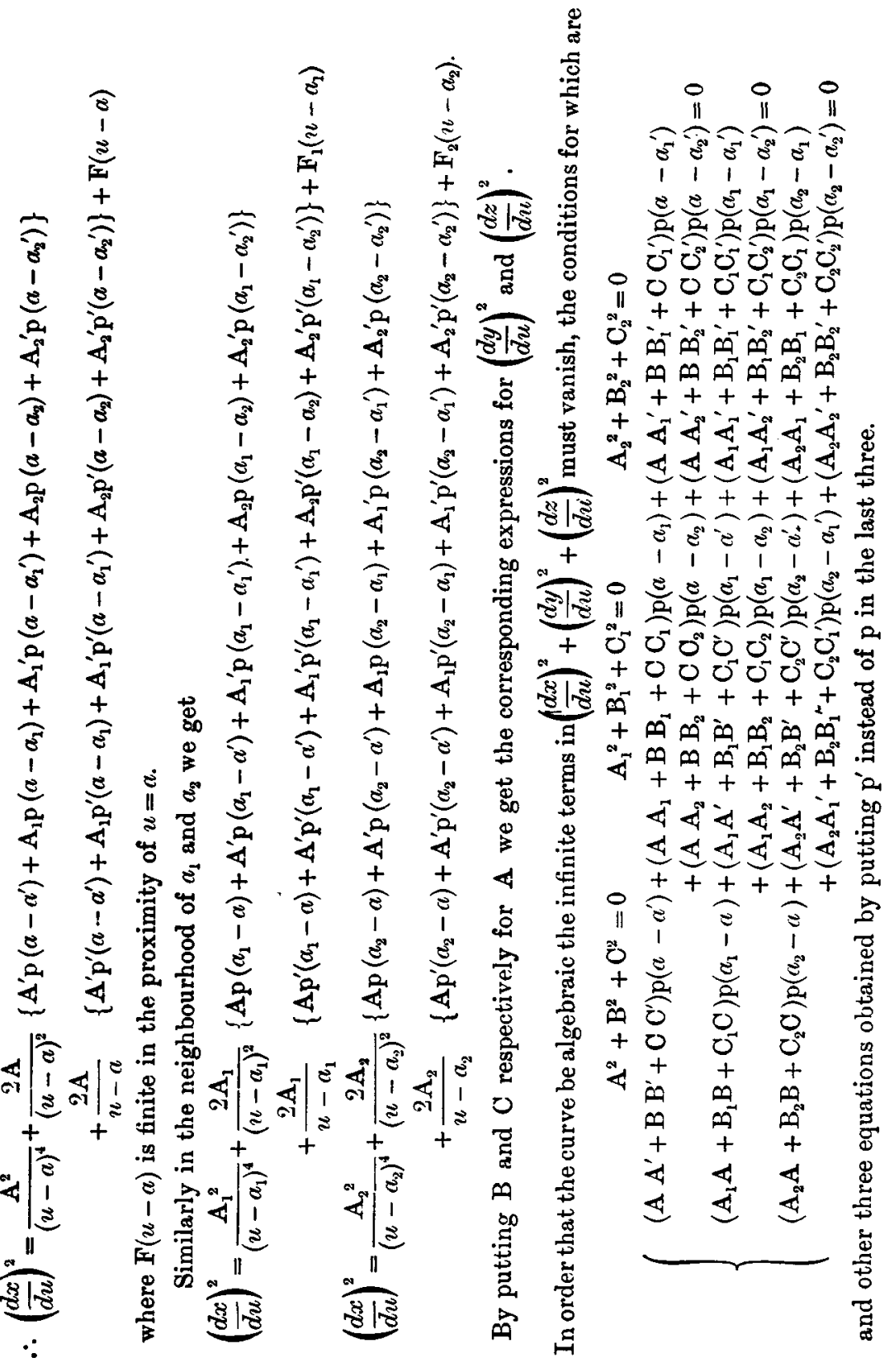


The values of $a, a_{1}, a_{2}, a^{\prime}, a_{1}^{\prime}, a_{2}^{\prime}$ we have already stated.

Hence there are nine equations of condition, and these can be fulfilled since we have nine quantities at our disposal, viz.,
A B C
$A_{1} B_{1} C_{3}$
$\mathrm{A}_{2} \mathrm{~B}_{2} \mathrm{C}_{2}$,

or their conjugates. So that corresponding to Kiepert's plane curve $r^{3}=\cos 3 \phi$ there exists a curve of double curvature whose arcs represent the elliptic integral of the first kind.

I shall prove the same thing by another method which will be found exceedingly useful in the next section.

$$
\begin{aligned}
& \frac{d x}{d u}=-\operatorname{Ap}(u-a)-\mathrm{A}^{\prime} \mathrm{p}\left(u-a^{\prime}\right)-\mathrm{A}_{1} \mathrm{p}\left(u-a_{1}\right)-\mathrm{A}_{1}^{\prime} \mathrm{p}\left(u-a_{1}{ }^{\prime}\right) \\
& -\mathrm{A}_{2} \mathrm{p}\left(u-a_{2}\right)-\mathrm{A}_{2}{ }^{\prime} \mathrm{p}\left(u-a_{2}{ }^{\prime}\right)
\end{aligned}
$$

For shortness write this as follows :

$$
\begin{aligned}
\frac{d x}{d u} & =-\mathrm{A} \mathrm{p}(u-a)-\mathrm{P}(u) \\
\text { or } \frac{d x}{d u} & =-\mathrm{A}_{1} \mathrm{p}\left(u-a_{1}\right)-\mathrm{P}_{1}(u) \\
\text { or } \frac{d x}{d u} & =-\mathrm{A}_{2} \mathrm{p}\left(u-a_{2}\right)-\mathrm{P}_{2}(u) .
\end{aligned}
$$

Similarly

$$
\begin{aligned}
& \frac{d y}{d u}=-\mathrm{Bp}(u-a)-\mathrm{Q}(u)=-\mathrm{B}_{1} \mathrm{p}\left(u-a_{1}\right)-\mathrm{Q}_{1}(u)=-\mathrm{B}_{2} \mathrm{p}\left(u-a_{2}\right)-\mathrm{Q}_{2}(u) \\
& \frac{d z}{d u}=-\mathrm{Cp}(u-a)-\mathrm{R}(u)=-\mathrm{C}_{1} \mathrm{p}\left(u-a_{1}\right)-\mathrm{R}_{1}(u)=-\mathrm{C}_{2} \mathrm{p}\left(u-a_{2}\right)-\mathrm{R}_{2}(u) .
\end{aligned}
$$

Then, in order to prove that the curve (1) is algebraic, we have to show that the conditions are satisfied that

$$
\left(\frac{d x}{d u}\right)^{2}+\left(\frac{d y}{d u}\right)^{2}+\left(\frac{d z}{d u}\right)^{2}
$$

have no infinite terms for $u=a, u=a_{1}$, or $u=a_{2}$.

To show this we have to develop

$$
\frac{d x}{d u}, \quad \frac{d y}{d u}, \quad \frac{d z}{d u}
$$

in the neighbourhood of the points $a, a_{1}$ and $a_{2}$. 
For the development of $\mathrm{p}(u-a)$ see Halphen's F.E., vol. I., p. 92, and for that of $\mathrm{P}(u)$ see footnote, p. 37 . Using these, we get for the proximity of $u=a$

(2) $\frac{d x}{d u}=-\frac{\mathrm{A}}{(u-a)^{2}}-\mathrm{A} c_{0}-\mathrm{A} c_{2}(u-a)^{2}-\ldots$

$$
\ldots-\mathrm{P}(a)-(u-a) \mathrm{P}^{\prime}(a)-\frac{(u-a)^{2}}{2} \mathrm{P}^{\prime \prime}(a)-\ldots
$$

Similarly for the neighbourhoods of $u=a_{1}$ or $u=a_{2}$ we get

$$
\begin{aligned}
\frac{d x}{d u}=- & \frac{\mathbf{A}_{1}}{\left(u-a_{1}\right)^{2}}-\mathbf{A}_{1} c_{0}-\mathbf{A}_{1} c_{2}\left(u-a_{1}\right)^{2}-\ldots \\
& \ldots-\mathbf{P}_{1}\left(a_{1}\right)-\left(u-a_{1}\right) \mathbf{P}_{1}^{\prime}\left(a_{1}\right)-\frac{\left(u-a_{1}\right)^{2}}{2} \mathbf{P}^{\prime \prime}\left(a_{1}\right)-\ldots \\
\frac{d x}{d u}=- & \frac{\mathbf{A}_{2}}{\left(u-a_{2}\right)^{2}}-\mathbf{A}_{2} c_{0}-\mathbf{A}_{2} c_{2}\left(u-a_{2}\right)^{2}-\ldots \\
& \ldots-\mathbf{P}_{2}\left(a_{2}\right)-\left(u-a_{2}\right) \mathbf{P}_{2}^{\prime}\left(a_{2}\right)-\frac{\left(u-a_{2}\right)^{2}}{2} \mathbf{P}^{\prime \prime}\left(a_{2}\right)-\ldots
\end{aligned}
$$

Squaring (2) we get

$$
\left(\frac{d x}{d u}\right)^{2}=\frac{\mathrm{A}^{2}}{(u-a)^{4}}+\frac{2 \mathrm{~A}}{(u-a)^{2}}\left[\mathrm{~A} c_{0}+\mathrm{P}(a)\right]+\frac{2 \mathrm{~A} \cdot \mathrm{P}^{\prime}(a)}{u-a}+\mathrm{F}(u-a)
$$

where $\mathrm{F}(u-a)$ is finite in the neighbourhood of $u=a$.

By putting $B$ and $C$ respectively in place of $A$ we get similar expressions for $\left(\frac{d y}{d u}\right)^{2}$ and $\left(\frac{d z}{d u}\right)^{2}$.

Then the necessary conditions that $\left(\frac{d x}{d u}\right)^{2}+\left(\frac{d y}{d u}\right)^{2}+\left(\frac{d z}{d u}\right)^{2}$

be not infinite for $u=a$ are (where $\Sigma$ denotes the sum of all similar terms got by interchanging $A, B$, and $C$, and $P, Q$, and $R$ )

$$
\begin{aligned}
& \Sigma \mathbf{A}^{2}=0 \\
& \Sigma \mathrm{A}\left[\mathrm{A} c_{0}+\mathrm{P}(a)\right]=0 \\
& \Sigma \mathbf{A} \cdot \mathbf{P}^{\prime}(a)=0
\end{aligned}
$$

or we may write them

(3) $\quad \Sigma \mathrm{A}^{2}=0, \quad \Sigma \mathrm{A} \cdot \mathrm{P}(a)=0 . \quad \Sigma \mathrm{A} \cdot \mathrm{P}^{\prime}(a)=0$ 
Similarly in the neighbourhood of $u=a_{1}$ and $u=a_{2}$ respectively we get

$$
\text { (5) } \quad \Sigma \mathbf{A}_{2}{ }^{2}=0, \quad \Sigma \mathbf{A}_{2} \cdot P_{2}\left(a_{2}\right)=0, \quad \Sigma \mathbf{A}_{2} \cdot P_{2}^{\prime}\left(a_{2}\right)=0 \text {. }
$$

Thus we have in (3) (4) and (5) altogether nine equations of condition to be fulfilled in order that the given curve be algebraic, and these can be satisfied since we have nine quantities at our disposal, viz., $A B C \quad A_{1} B_{1} C_{1} \quad A_{2} B_{2} C_{2}$ or their conjugates, and hence we come to the same conclusion as before.

\section{XII.}

Again, by Lilienthal's notation we can readily generalise Serret's plane curves and obtain a corresponding group of curves on a sphere.

We have seen (vide equation (16) p. 32 and footnote there) that for Serret's curves

$$
\begin{aligned}
\frac{d x+i d y}{d u}=\sum_{\nu=1}^{m} c_{1}, \nu \frac{d^{2 \nu+1}}{d u^{2 \nu+1}} \log \sigma\left(u-\frac{\omega}{2}+\frac{\omega^{\prime}}{2}\right) \\
+\sum_{\nu=1}^{n} c_{2, \nu} \frac{d^{2 \nu+1}}{d u^{2 \nu+1}} \log \left(u+\frac{\omega}{2}-\frac{\omega^{\prime}}{2}\right)
\end{aligned}
$$

i.e.,

$$
\text { (1) } x+i y=\sum_{\nu=1}^{m} c_{1, \nu} \frac{d^{2 \nu}}{d u^{2 \nu}} \log \sigma(u-a)+\sum_{\nu=1}^{n} c_{2, \nu} \frac{d^{2 \nu}}{d u^{2 \nu}} \log \sigma\left(u-a_{1}\right)
$$

where $a=\frac{\omega}{2}-\frac{\omega^{\prime}}{2}$, and $a_{1}=-\frac{\omega}{2}+\frac{\omega^{\prime}}{2}$.

The curves having obviously two infinities, $u=a$ and $u=a_{1}$, therefore writing the expressions for $x, y$, and $z$ in Lilienthal's notation $[c f . \mathrm{X} .(1)]$ we get 
(2) $x=\mathrm{A} \mathrm{p}(u-a)+\mathrm{A}_{2} \mathrm{p}^{\prime \prime}(u-a)+\ldots \ldots+\mathrm{A}_{2 \nu} \quad \mathrm{p}^{(2 \nu)}(u-a)$

$$
\begin{aligned}
& +\mathrm{A}^{\prime} \mathrm{p}\left(u-a^{\prime}\right)+\mathrm{A}_{2}^{\prime} \mathrm{p}^{\prime \prime}\left(u-a^{\prime}\right)+\ldots+\mathrm{A}_{2 \nu}^{\prime} \mathrm{p}^{(2 \nu)}\left(u-a^{\prime}\right) \\
& +\mathrm{A}_{1} \mathrm{p}\left(u-a_{1}\right)+\mathrm{A}_{3} \mathrm{p}^{\prime \prime}\left(u-a_{1}\right)+\ldots+\mathrm{A}_{2 \nu+1} \mathrm{p}^{(2 \nu)}\left(u-a_{1}\right) \\
& +\mathrm{A}_{1}^{\prime} \mathrm{p}\left(u-a_{1}\right)+\mathrm{A}_{3}^{\prime} \mathrm{p}^{\prime \prime}\left(u-a_{1}^{\prime}\right)+\ldots \ldots+\mathrm{A}_{2 \nu+1}^{\prime} \mathrm{p}^{(2 \nu)}\left(u-a_{1}^{\prime}\right)
\end{aligned}
$$

with similar expressions for $y$ and $z$ by putting $B$ and $C$ respectively in place of $A$.

For shortness, write the above

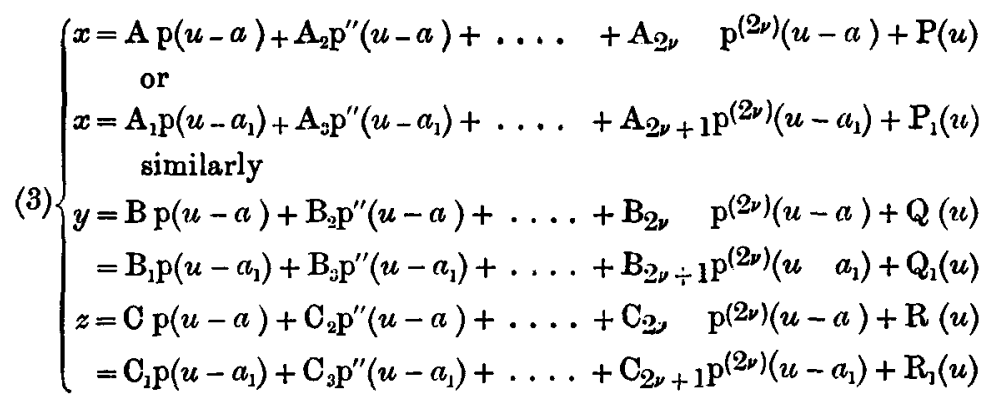

Comparing this abbreviated notation with the full expression for $x$ given above, it is evident that $P Q R P_{1} Q_{1} R_{1}$ have only even derivatives.

Then, just as in the preceding sections, in order to prove that the curves for (3) are algebraic we have to show that the conditions are satisfied that

$$
\left(\frac{d x}{d u}\right)^{2}+\left(\frac{d y}{d u}\right)^{2}+\left(\frac{d z}{d u}\right)^{2}
$$

be constant for $u=a$, or $u=a$.

Now,

$$
\begin{aligned}
\frac{d x}{d u}= & \mathrm{Ap}^{\prime}(u-a)+\mathrm{A}_{2} \mathrm{p}^{\prime \prime \prime}(u-a)+\ldots \\
& \ldots+\mathrm{A}_{2 \nu-2} \mathrm{p}^{(2 \nu-1)}(u-a)+\mathrm{A}_{2 \nu} \mathrm{p}^{(2 \nu+1)}(u-a)+\mathrm{P}^{\prime}(u) .
\end{aligned}
$$

We have to develop this in the neighbourhood of $u=a$. 
But

$$
\begin{aligned}
& \mathrm{p} u=\frac{1}{u^{2}}+c_{0}+c_{2} u^{2}+c_{4} u^{4}+c_{6} u^{6}+c_{8} u^{8}+\ldots \\
& \because \mathrm{p}^{\prime} u=-\frac{1.2}{u^{3}} \\
& \text { (vide Halphen's F. E., vol. I. p. 92) } \\
& \mathrm{p}^{\prime \prime} u=\frac{1.2 .3}{u^{4}} \quad+2 c_{2}+12 c_{4} u^{2}+30 c_{6} u^{4}+56 c_{8} u^{\mathrm{B}}+\ldots \\
& p^{\prime \prime \prime} u=-\frac{1.2 .3 .4}{u^{5}} \quad+24 c_{4} u+120 c_{6} u^{3}+336 c_{8} u^{5}+\ldots \\
& \mathrm{p}^{\mathrm{Iv}} u=\frac{1.2 .3 .4 .5}{u^{6}} \quad+24 c_{4}+360 c_{6} u^{2}+1680 c_{8} u^{4}+\ldots \\
& \mathrm{p}^{\mathrm{v}} u=-\frac{1.2 .3 .4 .5 .6}{u^{7}} \quad+720 c_{8} u+6720 c_{8} u^{3}+\ldots
\end{aligned}
$$

We may therefore include the even and odd derivatives of pu under the following useful general formula:-

$$
\begin{aligned}
& \mathrm{p}^{(2 r)} u=\frac{(2 r+1) !}{u^{2 r+2}}+c_{0,2 r}+c_{2,2 r} u^{2}+c_{4,2 r} u^{4}+\ldots \\
& \mathrm{p}^{(2 r+1)} u=-\frac{(2 r+2) !}{u^{2 r+3}}+c_{1,2 r+1} u+c_{3,2 r+1} u^{3}+c_{3,2 r+1} u^{5}+\ldots .
\end{aligned}
$$

Using the latter for the development of $\frac{d x}{d u}$ above, we get

(4) $\frac{d x}{d u}=-\frac{2 \mathrm{~A}}{(u-a)^{3}}-\frac{4 ! \mathrm{A}_{2}}{(u-a)^{5}}-\ldots-\frac{(2 v) ! \mathrm{A}_{2 y-2}}{(u-a)^{2 v+1}}-\frac{(2 v+2) ! \mathrm{A}_{2 v}}{(u-a)^{2 v+3}}$

$$
\begin{aligned}
& +\left(c_{1,1} \mathrm{~A}+c_{1,3} \mathrm{~A}_{2}+\ldots+c_{1,2 v+1} \mathrm{~A}_{2 v}\right)(u-a) \\
& +\left(c_{3,1} \mathrm{~A}+c_{3,3} \mathrm{~A}_{2}+\ldots+c_{3,2 v+1} \mathrm{~A}_{2 y}\right)(u-a)^{3} \\
& +\left(c_{2 y+1,1} \mathbf{A}+c_{2 y+1,9} \mathbf{A}_{2}+\ldots+c_{2 y+1,2 y+1} \mathbf{A}_{2 \nu}\right)(u-a)^{2 \nu+1} \\
& +(u-a) \mathrm{P}^{\prime \prime}(a)+\frac{(u-a)^{3}}{3 !} \mathrm{P}^{\mathrm{IV}}(a)+\ldots
\end{aligned}
$$

$$
\ldots+\frac{(u-a)^{2 v+1}}{(2 v+1) !} \mathrm{P}^{(2 y+2)}(a) .
$$

* For the development of $\mathrm{P}^{\prime}(u)$ in the proximity of $u=a, c f$. footnote, $\mathrm{p}$. 37, keeping in mind that $\mathrm{P}(u)$ has only even derivatives as already pointed out. 
Similarly, for the neighbourhood of $u=a_{1}$, we get

(5) $\frac{d x}{d u}=-\frac{2 \mathrm{~A}_{1}}{\left(u-a_{1}\right)^{3}}-\frac{4 ! \mathrm{A}_{3}}{\left(u-a_{1}\right)^{5}}-\cdots-\frac{(2 v) ! \mathrm{A}_{2 \nu-1}}{\left(u-a_{1}\right)^{2 v+1}}-\frac{(2 v+2) ! \mathrm{A}_{2 v+1}}{\left(u-a_{1}\right)^{2 v+3}}$

$$
\begin{aligned}
& +\left(c_{1,1} A_{1}+c_{1,8} A_{3}+\ldots+c_{1,2 v+1} A_{2 v+1}\right)\left(u-a_{1}\right) \\
& +\left(c_{3,1} A_{1}+c_{3,3} A_{3}+\ldots+c_{3,2 y+1} A_{2 y+1}\right)\left(u-a_{1}\right)^{3} \\
& +\left(c_{2 y+1,1} A_{1}+c_{2 y+1,3} A_{3}+\ldots+c_{2 y+1,2 y+1} A_{2 y+1}\right)\left(u-a_{1}\right)^{2 y+1} \\
& +\left(u-a_{1}\right) \mathbf{P}_{1}^{\prime \prime}\left(a_{1}\right)+\frac{\left(u-a_{1}\right)^{3}}{3 !} \mathbf{P}_{1}{ }^{\mathrm{vV}}\left(a_{1}\right)+\ldots \\
& \ldots+\frac{\left(u-a_{1}\right)^{2 v+1}}{(2 v+1) !} \mathbf{P}_{1}^{(2 v+2)}\left(a_{1}\right) .
\end{aligned}
$$

On replacing $A$ by $B$ and $O$ respectively, we get the corresponding expressions for $\frac{d y}{d u}$ and $\frac{d z}{d u}$.

Now, in order that the condition

$$
\left(\frac{d x}{d u}\right)^{2}+\left(\frac{d y}{d u}\right)^{2}+\left(\frac{d z}{d u}\right)^{2}=\text { constant }
$$

be fulfilled in the neighbourhood of $u=a$, and $u=a_{1}$, it is of course necessary that the terms containing $u-a$ and $u-a_{1}$ in the denominator vanish. On squaring (4) and (5) we see that the necessary conditions for this are (where $\Sigma$ denotes the sum of all similar terms got by interchanging $A, B$, and $C$, and $P, Q$, and $R$ )

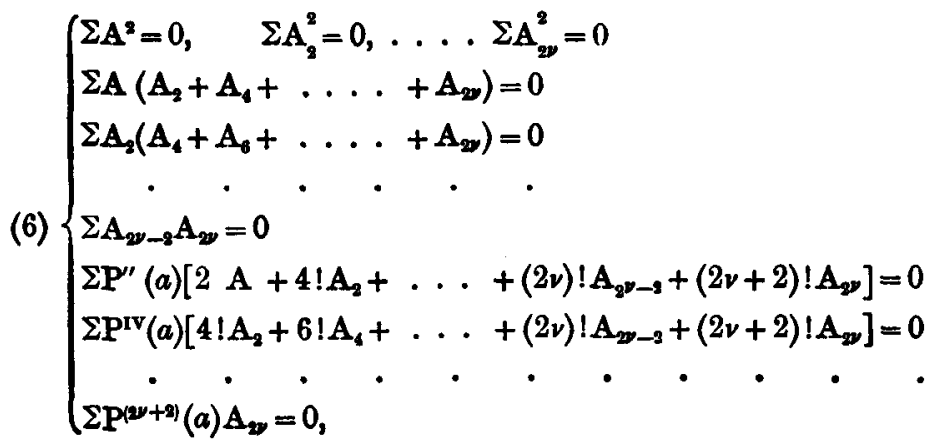


and similar equations to be satisfied for the neighbourhood of $u=a_{1}$. But from (2) we see that

(7) $\frac{A_{2}}{A}=\frac{B_{2}}{B}=\frac{C_{2}}{C}=\lambda$, say; and $\frac{A_{4}}{A_{2}}=\frac{B_{4}}{B_{2}}=\frac{C_{4}}{C_{\varepsilon}}$, and so on.

Hence we may put

$$
\begin{cases}\mathrm{A}_{2}=\lambda \mathrm{A}, & \mathrm{A}_{4}=\lambda_{1} \mathrm{~A} \ldots \ldots \mathrm{A}_{2 y}=\lambda_{\nu-1} \mathrm{~A} . \\ \mathrm{B}_{2}=\lambda \mathrm{B}, & \mathrm{B}_{4}=\lambda_{1} \mathrm{~B} \ldots \mathrm{B}_{2 \nu}=\lambda_{\nu-1} \mathrm{~B} . \\ \mathrm{C}_{2}=\lambda \mathrm{C}, & \mathrm{C}_{4}=\lambda_{1} \mathrm{C} \ldots \mathrm{C}_{2 \nu}=\lambda_{\nu-1} \mathrm{C} .\end{cases}
$$

Therefore (6) reduces to

(9) $\Sigma \mathrm{A}^{2}=0, \quad \Sigma \mathrm{A} \cdot \mathrm{P}^{\prime \prime}(a)=0, \quad \Sigma \mathrm{A} \cdot \mathrm{P}^{\mathrm{iv}}(a)=0, \ldots \Sigma \mathrm{A} \cdot \mathrm{P}^{(2 \nu+2)}(a)=0$.

Similarly, for the neighbourhood of $u=a_{1}$, on putting

$$
\begin{cases}\mathbf{A}_{3}=\mu \mathrm{A}_{1}, & \mathbf{A}_{5}=\mu_{1} \mathrm{~A}_{1} \ldots \mathrm{A}_{2 \nu+1}=\mu_{\nu-1} \mathrm{~A}_{1} \\ \mathbf{B}_{3}=\mu \mathrm{B}_{1}, & \mathbf{B}_{5}=\mu_{1} \mathrm{~B}_{1} \ldots \mathrm{B}_{2 \nu+1}=\mu_{\nu-1} \mathrm{~B}_{1} \\ \mathrm{C}_{3}=\mu \mathrm{C}_{1}, & \mathbf{C}_{5}=\mu_{1} \mathrm{C}_{1} \ldots \mathrm{C}_{2 \nu+1}=\mu_{\nu-1} \mathrm{C}_{1}\end{cases}
$$

we get

(11) $\Sigma \mathbf{A}_{1}^{2}=0, \Sigma \mathbf{A}_{1} \cdot P_{1}^{\prime \prime}\left(a_{1}\right)=0, \Sigma A_{1} \cdot P_{1}^{I V}\left(a_{1}\right)=0 \ldots \Sigma A_{1} \cdot P_{1}^{(2 \nu+2)}\left(a_{1}\right)=0$

We thus have in (9) and (11) altogether $2 v+4$ equations among $2 v+6$ quantities

$$
A B C \quad A_{1} B_{1} C_{1} \quad \lambda \lambda_{1} \ldots \lambda_{\nu-1} \mu \mu_{1} \ldots \mu_{\nu-1} .
$$

But if we further stipulate that the curves be spherical ones, this is equivalent to other two conditions, for, proceeding in the same way as Lilienthal does for the spherical lemniscate, we have to find the conditions that the expression $(x-\alpha)^{2}+(y-\beta)^{2}+(z-\gamma)^{2}$ be constant, i.e., have no infinities for $u=a, u=a_{1}$.

Now, developing in the proximity of $u=a$ the value of $x$ given in (3) we get ( $f f$. footnote, p. 37, and equation (4) above)

$$
\begin{aligned}
x= & +\frac{\mathbf{A}}{(u-a)^{2}}+\frac{3 ! \mathbf{A}_{2}}{(u-a)^{4}}+\ldots .+\frac{(2 v+1) ! \mathrm{A}_{2 v}}{(u-a)^{2 \nu+2}} \ldots . \\
& +\mathrm{P}(a)+\frac{(u-a)^{2} \mathrm{P}^{\prime \prime}(a)}{2}+\ldots .+\frac{(u-a)^{2 \nu} \mathrm{P}^{(2 \nu)}(a)}{(2 v) !} .
\end{aligned}
$$


Wherefore, keeping (8) in mind, we have

$$
\begin{aligned}
x-a= & +\frac{\mathrm{A}}{(u-a)^{2}}+\frac{3 ! \lambda \mathrm{A}}{(u-a)^{4}}+\ldots+\frac{(2 v+1) ! \lambda_{\nu-1} \mathrm{~A}}{(u-a)^{2 \nu+2}} \ldots \\
& +\mathrm{P}(a)-a+\frac{(u-a)^{2} \mathrm{P}^{\prime \prime}(a)}{2}+\ldots \ldots+\frac{(u-a)^{2 \nu} \mathrm{P}^{(2 \nu)}(a)}{(2 v) !} .
\end{aligned}
$$

Similarly, in the neighbourhood of $u=a_{1}$

$$
\begin{aligned}
x-a= & +\frac{A_{1}}{\left(u-a_{1}\right)^{2}}+\frac{3 ! \mu \mathrm{A}_{1}}{\left(u-a_{1}\right)^{4}}+\ldots+\frac{(2 \nu+1) ! \mu_{\nu-1} \mathrm{~A}_{1}}{\left(u-a_{1}\right)^{2 \nu+2}} \ldots \\
& +\mathrm{P}_{1}\left(a_{1}\right)-\alpha+\frac{\left(u-a_{1}\right)^{2} \mathrm{P}_{1}^{\prime \prime}\left(a_{1}\right)}{2}+\ldots+\frac{\left(u-a_{1}\right)^{2} \nu \mathrm{P}^{(2 \nu)}\left(a_{1}\right)}{(2 v) !} .
\end{aligned}
$$

We get the corresponding expressions for $y-\beta$ and $z-\gamma$ on replacing $A$ by $B$ and $C$ respectively.

Then, on squaring, we find that in order that the curve be spherical, i.e., in order that $(x-\alpha)^{2}+(1 y-\beta)^{2}+(z-\gamma)^{2}$ have no infinities at $u=a$ and $u=a_{1}$, the terms containing $u-a$ and $u-a_{1}$ in the denominator must vanish.

Therefore, in addition to equations of the types in (9) and (11) we also get

$$
\left\{\begin{array}{l}
\mathrm{A}[\mathrm{P}(a)-a]+\mathrm{B}[\mathrm{Q}(a)-\beta]+\mathrm{C}[\mathrm{R}(a)-\gamma]=0 \\
\mathrm{~A}_{1}\left[\mathrm{P}_{1}\left(a_{1}\right)-a\right]+\mathrm{B}_{1}\left[\mathrm{Q}_{1}\left(a_{1}\right)-\beta\right]+\mathrm{C}_{1}\left[\mathrm{R}_{1}\left(a_{1}\right)-\gamma\right]=0 .
\end{array}\right.
$$

Hence we have altogether $2 v+6$ equations of condition and and $2 v+6$ quantities at our disposal, so that the necessary conditions can be satisfied that, corresponding to Serret's infinite group of plane curves we have an infinite group of spherical curves whose arcs are elliptic integrals of the first kind.

Finally, I wish to prove that corresponding to Kiepert's infinity of plane curves there is an infinity of spherical ones whose arcs represent the first elliptic integral.

The equation for Kiepert's curves is (see p. 29)

$$
\frac{d x+i d y}{d u}=\sum_{\nu=1}^{r_{1}} c_{1, \nu} \frac{d^{\nu+1} \log \sigma\left(u-a_{1}\right)}{d u^{\nu+1}}+\ldots+\sum_{\nu=1}^{r_{m}} c_{m, \nu} \frac{d \nu+1 \log \sigma\left(u-a_{m}\right)}{d u^{\nu+1}}
$$


i.e.,

(1a) $x+i y=\sum_{\nu=1}^{r_{1}} c_{1, \nu} \frac{d^{\nu} \log \sigma\left(u-a_{1}\right)}{d u^{\nu}}+\ldots+\sum_{\nu=1}^{v_{m}} c_{m, \nu} \frac{d^{\nu} \log \sigma\left(u-a_{m}\right)}{d u^{\nu}}$

where the infinities $u=a_{1}, a_{2}, \ldots \ldots a_{m}$ have any given values.

We shall call the equations used $\left(1^{2}\right),\left(2^{a}\right)$, etc., to indicate that they are the analagous ones to (1), (2), etc., in the previous part of the section.

Since the points at infinity are $u=a_{1}, a_{2}, \ldots \ldots a_{m}$ we may represent the coordinates of Kiepert's curves thus :-

$$
\begin{aligned}
& \text { (2a) } x= \\
& \mathrm{A}_{1,0} \mathrm{p}\left(u-a_{1}\right)+\mathrm{A}_{1,1} \mathrm{p}^{\prime}\left(u-a_{1}\right)+\ldots+\mathbf{A}_{1, \nu} \mathrm{p}^{(\nu)}\left(u-a_{1}\right) \\
& +\mathrm{A}_{1, \mathrm{\nu}}^{\prime} \mathrm{p}\left(u-a_{1}^{\prime}\right)+\mathrm{A}_{1,1}^{\prime} \mathrm{p}^{\prime}\left(u-a_{1}^{\prime}\right)+\ldots+\mathrm{A}_{1, \nu}^{\prime} \mathrm{p}^{(\nu)}\left(u-a_{1}^{\prime}\right) \\
& +\mathrm{A}_{2, \mathrm{o}} \mathrm{p}\left(u-a_{2}\right)+\mathrm{A}_{2,1} \mathrm{p}^{\prime}\left(u-a_{2}\right)+\ldots+\mathrm{A}_{2, \nu} \mathrm{p}^{(v)}\left(u-a_{2}\right) \\
& +\mathrm{A}_{2,0}^{\prime} \mathrm{p}\left(u-a_{2}^{\prime}\right)+\mathrm{A}_{2,1}^{\prime} \mathrm{p}^{\prime}\left(u-a_{2}^{\prime}\right)+\ldots+\mathrm{A}_{2, \nu}^{\prime} \mathrm{p}^{(\nu)}\left(u-a_{2}^{\prime}\right) \\
& +\mathrm{A}_{m, 0} \mathrm{p}\left(u-a_{m}\right)+\mathrm{A}_{m, 1} \mathrm{p}^{\prime}\left(u-a_{m}\right)+\ldots+\mathrm{A}_{m, \nu} \mathrm{p}^{(v)}\left(u-a_{m}\right) \\
& +\mathbf{A}_{m, 0}^{\prime} \mathrm{p}\left(u-a_{m}^{\prime}\right)+\mathbf{A}_{m, 1}^{\prime} \mathrm{p}^{\prime}\left(u-a_{m}^{\prime}\right)+\ldots+\mathbf{A}_{m, \nu}^{\prime} \mathrm{p}^{(\nu)}\left(u-a_{m}^{\prime}\right)
\end{aligned}
$$

and similar expressions for $y$ and $z$ by putting $B$ and $C$ respectively for $\mathbf{A}$.

The contracted notation corresponding to (3) will be

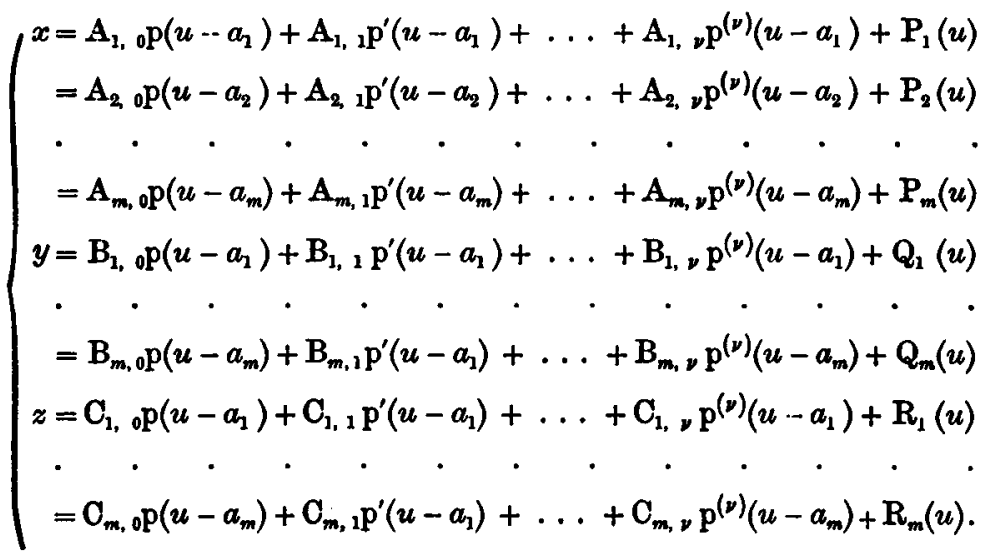


Therefore

$$
\begin{gathered}
\frac{d x}{d u}=\mathrm{A}_{1, \mathrm{p}} \mathrm{p}^{\prime}\left(u-a_{1}\right)+\mathrm{A}_{1,1} \mathrm{p}^{\prime \prime}\left(u-a_{1}\right)+\ldots \\
\ldots+\mathrm{A}_{1, \nu-1} \mathrm{p}^{(\nu)}\left(u-a_{1}\right)+\mathrm{A}_{1, \nu} \mathrm{p}^{(\nu+1)}\left(u-a_{1}\right)+\mathrm{P}_{1}^{\prime}(u) .
\end{gathered}
$$

Developing this in the neighbourhood of $u=a_{1}$ we get, since the derivatives are alternately odd and even (vide the general expressions for the odd and even derivatives of $\mathrm{p} u$ on p. 56),

$$
\begin{aligned}
& \text { (4a) } \frac{d x}{d u}=-\frac{2 \mathbf{A}_{1,0}}{\left(u-a_{1}\right)^{3}}+\frac{3 ! \mathbf{A}_{1,1}}{\left(u-a_{1}\right)^{4}}-\ldots+\frac{(\nu+1) ! \mathbf{A}_{1, \nu-1}}{\left(u-a_{1}\right)^{\nu+2}}-\frac{(\nu+2) ! \mathbf{A}_{1, \nu}}{\left(u-a_{1}\right)^{\nu+3}} * \\
& +\left(c_{0,2} \mathrm{~A}_{1,1}+c_{0,4} \mathrm{~A}_{1,3}+\ldots+c_{0, \nu} \mathrm{A}_{1, \nu-1}\right) \\
& +\left(c_{2,3} A_{1,1}+c_{2,4} A_{1,3}+\ldots+c_{2, \nu} A_{1, \nu-1}\right)\left(u-a_{1}\right)^{2} \\
& +\left(c_{\nu, 2} \mathrm{~A}_{1,1}+c_{\nu, 4} \mathrm{~A}_{1,3}+\ldots+c_{\nu, \nu} \mathrm{A}_{1, \nu-1}\right)\left(u-a_{1}\right)^{\nu} \\
& +\left(c_{1,1} A_{1,0}+c_{1,3} A_{1,2}+\ldots+c_{1, \nu+1} A_{1, v}\right)\left(u-a_{1}\right) \\
& +\left(c_{3,1} A_{1,0}+c_{3,3} A_{1,2}+\cdots+c_{3, \nu+1} A_{1, \nu}\right)\left(u-a_{1}\right)^{3} \\
& +\left(c_{\nu+1,1} \mathrm{~A}_{1,0}+c_{\nu+1,3} \mathrm{~A}_{1,2}+\ldots+c_{\nu+2, \nu+1} \mathrm{~A}_{1, \nu}\right)\left(u-a_{3}\right)^{\nu+1} \\
& +\mathrm{P}_{1}^{\prime}\left(a_{1}\right)+\left(u-a_{1}\right) \mathrm{P}_{1}^{\prime \prime}\left(a_{1}\right)+\frac{\left(u-a_{1}\right)^{2}}{2} \mathbf{P}_{1}^{\prime \prime \prime}\left(a_{1}\right) \ldots \\
& \ldots+\frac{\left(u-a_{1}\right)^{\nu}}{\nu !} P_{1}^{(\nu+1)}\left(a_{1}\right)
\end{aligned}
$$

and corresponding expressions for $\frac{d x}{d u}$ in the neighbourhoods of

$$
u=a_{2}, u=a_{3}, \ldots u=a_{m} .
$$

Also, putting $B$ and $C$ respectively in place of $A$ we get similar expansions for $\frac{d y}{d u}$ and $\frac{d z}{d u}$.

Then expressing the conditions that $\left(\frac{d x}{d u}\right)^{2}+\left(\frac{d y}{d u}\right)^{2}+\left(\frac{d z}{d u}\right)^{2}$

Taking for granted that $\nu$ is even; of course it does not matter whether it is odd or even-it is merely a question of which sign we shall use. 
may have no infinities at $u=a_{1}$ we get

(6a)

$$
\begin{aligned}
& \left(\begin{array}{l}
\Sigma A_{1,0}^{2}=0, \Sigma A_{1,1}^{2}=0, \Sigma A_{1,2}^{2}=0, \ldots A_{1, \nu}^{2}=0 \\
\Sigma A_{1,0}\left(A_{1,1}+A_{1,2}+\cdots+A_{1, \nu}\right)=0
\end{array}\right. \\
& \Sigma A_{1,1}\left(A_{1,2}+A_{1,3}+\ldots+A_{1, \nu}\right)=0 \\
& \mathrm{SA}_{1, \nu-1} \mathbf{A}_{1, \nu}=0 \\
& \Sigma \mathrm{P}_{1}^{\prime}\left(a_{1}\right)\left[-2 \mathrm{~A}_{1,0}+3 ! \mathrm{A}_{1,1}-4 ! \mathrm{A}_{1,2}+\cdots-(\nu+2) ! \mathrm{A}_{1, \nu}\right]=0 \\
& \Sigma \mathbf{P}_{1}^{\prime \prime}\left(a_{1}\right)\left[-2 \mathbf{A}_{1,0}+3 ! \mathbf{A}_{1,1}-4 ! \mathbf{A}_{1,2}+\cdots-(\nu+2) ! \mathbf{A}_{1, \nu}\right]=0 \\
& \Sigma P_{,}^{\prime \prime \prime}\left(a_{1}\right)\left[-2 \Lambda_{1,0}+3 ! A_{1,1}-4 ! A_{1,2}+\cdots-(\nu+2) ! A_{1, \nu}\right]=0 \\
& \Sigma \mathrm{P}_{1}^{\mathrm{IV}}\left(a_{1}\right)\left[+3 ! \mathrm{A}_{1,1}-4 ! \mathrm{A}_{1,2} \quad+\cdots-(v+2) ! \mathrm{A}_{1, \nu}\right]=0 \\
& \Sigma \mathbf{P}_{1} \mathbf{v}\left(a_{1}\right)\left[-4 ! A_{1,2}+5 ! A_{13} \quad-\cdots-(v+2) ! A_{1, \nu}\right]=0 \\
& \Sigma \mathrm{P}_{1}^{(\nu+1)}\left(a_{1}\right) \cdot \mathrm{A}_{1, \nu}=0
\end{aligned}
$$

But

(7a) $\frac{A_{1,1}}{A_{1,0}}=\frac{B_{1,1}}{B_{1,0}}=\frac{C_{1,1}}{C_{1,0}}=\lambda_{10}$, say; and $\frac{A_{1,2}}{A_{1,1}}=\frac{B_{1,2}}{B_{1,1}}=\frac{C_{1,2}}{C_{1,1}}$,

and so on.

Hence we may put

$$
\begin{cases}A_{1,1}=\lambda_{1,1} A_{1,0}, & A_{1,2}=\lambda_{1,2} A_{1,0} \ldots \ldots . A_{1, \nu}=\lambda_{1, \nu} A_{1,0} \\ B_{1,1}=\lambda_{1,1} B_{1,0}, & B_{1,2}=\lambda_{1,2} B_{1,0}, \ldots . B_{1, \nu}=\lambda_{1, \nu} B_{1,0} \\ C_{1,1}=\lambda_{1,1} C_{1,0}, & C_{1,2}=\lambda_{1,2} C_{1,0}, \ldots \ldots, C_{1, \nu}=\lambda_{1, \nu} C_{1,0} .\end{cases}
$$

Therefore $(6 a)$ reduces to

$$
\begin{aligned}
& \left(\Sigma A_{1,0}^{2}=0, \quad \Sigma A_{1,0} P_{1}^{\prime}\left(a_{1}\right)=0, \quad \Sigma A_{1,0} P_{1}^{\prime \prime}\left(a_{1}\right)=0 \ldots \Sigma A_{1,0} \mathbf{P}_{1}^{(\nu+1)}\left(a_{1}\right)=0\right. \text {. } \\
& \text { Similarly for the points } u=a_{2}, u=a_{3}, \ldots u=a_{m} \text {, we get } \\
& \left\{\mathrm{A}_{2,0}^{2}=0, \quad \Sigma \mathrm{A}_{2,0} \mathbf{P}_{2}^{\prime}\left(a_{2}\right)=0, \ldots \ldots \mathrm{\Sigma}_{2,0} \mathbf{P}_{2}^{(\nu+1)}\left(a_{2}\right)=0\right. \\
& \left\{\mathbf{A}_{3,0}^{2}=0, \quad \Sigma \mathbf{A}_{3,0} \mathbf{P}_{8}^{\prime}\left(a_{3}\right)=0, \ldots \ldots \mathbf{A}_{3,0} \mathbf{P}_{3}^{(\nu+1)}\left(a_{3}\right)=0\right. \\
& \Sigma \mathrm{A}_{m, 0}^{2}=0, \quad \Sigma \mathrm{A}_{m, 0} \mathrm{P}_{m}^{\prime}\left(a_{m}\right)=0, \quad \ldots \Sigma_{\mathrm{A}_{m, 0}} \mathrm{P}_{m}^{(\nu+2)}\left(a_{m}\right)=0
\end{aligned}
$$


In order that the curves be algebraic we thus have to satisfy the above $(v+2) m$ equations of condition among the $(v+3) m$ quantities :-

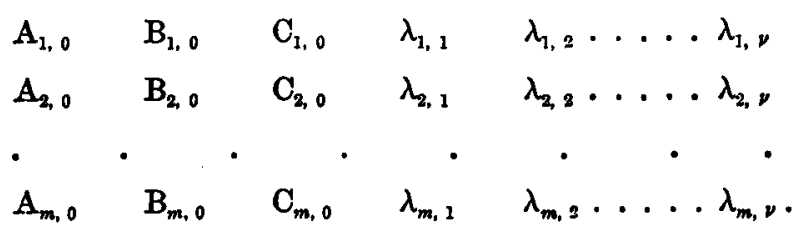

But if the curves be spherical ones this will introduce other $m$ conditions corresponding to (12) viz.,

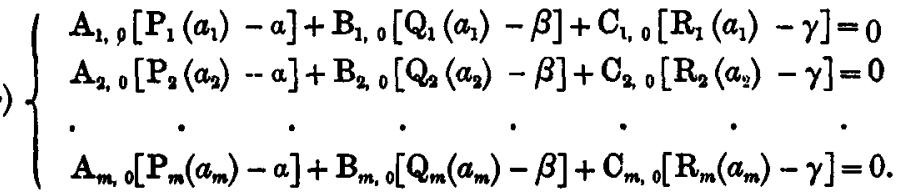

We have thus altogether $(\nu+3) m$ equations of condition and these can be satisfied as we have $(v+3) m$ quantities at our disposal, so that we have an infinity of algebraic spherical curves similar to Kiepert's plane curves and whose arcs likewise represent the elliptic integral of the first kind.

Kiepert asserted that to all the curves of single curvature whose arcs are elliptic integrals of the first kind there exist analogous curves of double curvature whose arcs are also first elliptic transcendentals. He says (De curvis quarum arcus, etc., p. 23), "omnes illæ curvæ, quæ in plano possunt investigari, singulares tantum casus sunt curvarum duplicis curvature." He only proved his assertion, however, in the particular case of the lemniscate which Roberts had done about 30 years before, and even to that case he could not apply the method he had discovered for plane curves. We now see that by generalising Lilienthal's method for 
plane curves, the feature of which is that it takes into account the behaviour of a curve at its singular points, we can prove the complete truth of Kiepert's statement. I have shown this in the case of the curve $r^{3}=\cos 3 \phi$ and, indeed, of Kiepert's plane curves in general which include Serret's infinity of curves and all the others as particular cases. These results, so far as I know, have not been obtained before, and they seem to be of considerable importance as they supply what was awanting in order to complete the geometrical representation of elliptic integrals of the first kind. 